Aguinel José Bastian Júnior

Validação externa de nomograma brasileiro para predição de câncer de próstata órgão-confinado em instituição terciária de ensino. Nomogramas da USP 
Aguinel José Bastian Júnior

\title{
Validação externa de nomograma brasileiro para predição de câncer de próstata órgão-confinado em instituição terciária de ensino. Nomogramas da USP
}

\author{
Tese apresentada à Faculdade de Medicina da \\ Universidade de São Paulo para obtenção do título \\ de Doutor em Ciências \\ Programa de: Urologia \\ Orientador: Prof. Dr. Marcos Francisco Dall'Oglio
}

São Paulo 
Dados Internacionais de Catalogação na Publicação (CIP)

Preparada pela Biblioteca da

Faculdade de Medicina da Universidade de São Paulo

Creprodução autorizada pelo autor

Bastian Júnior, Aguinel José

Validação externa de nomograma brasileiro para predição de câncer de próstata órgão-confinado em instituição terciária de ensino. Nomogramas da USP / Aguinel José Bastian Júnior. -- São Paulo, 2011.

Tese(doutorado)--Faculdade de Medicina da Universidade de São Paulo.

Programa de Urologia.

Orientador: Marcos Francisco Dall'Oglio .

Descritores: 1.Neoplasias da próstata 2.Prostatectomia 3.Modelos logísticos 4.Análise de regressão 5.Nomogramas

USP/FM/DBD-332/11 


\section{Dedicatória}

"Descubrir lo desconocido no es una especialidad de Simbad, de Erico el Rojo o de Copérnico. No hay un solo hombre que no sea un descubridor. Empieza descubriendo lo amargo, lo salado, lo cóncavo, lo liso, lo áspero, los siete colores del arco y los veintitantas letras del alfabeto; pasa por los rostros, los mapas, los animales y los astros; concluye por la duda o por la fe y por la certidumbre casi total de su propia ignorancia."

Borges

Dedico essa tese aos que descobrem e aos que, deslumbrados pela fronteira com o desconhecido, perseveram e se aventuram em direção ao novo, mesmo correndo o risco da incompreensão. 


\section{Agradecimentos}

Ao Prof. Dr. Miguel Srougi, incentivador e exemplo de academicismo médico de primeira grandeza. A urologia sob seu mando e égide alcança uma dimensão singular. Seu estilo está em tudo e sua influência é clara na postura e no trabalho das pessoas.

Ao Prof. Dr. Homero Bruschini, zeloso mentor da pós-graduação da disciplina de urologia da USP. Sua competência define os rumos exitosos desse programa, com público reconhecimento por parte da CAPES e da sociedade urológica.

Ao meu orientador, Prof. Dr. Marcos Francisco Dall'Oglio, oportunizador e exemplo de condução e comprometimento com seus pacientes e subordinados. Sua maneira incansável de abraçar novas causas e desafios inspira seus orientandos.

Ao amigo, Dr. Alexandre Crippa Sant'Anna, que desbravou o conhecimento aqui estendido ao desenvolver o primeiro nomograma brasileiro relacionado ao câncer de próstata.

Ao meu mestre e amigo, Prof. Dr. Getúlio Rodrigues de Oliveira Filho, exemplo de conduta científica e devoção ao ensino. Pessoa que me impressionou na seara didática, dada sua paixão inconteste por ensinar as criaturas, em especial as que trabalham com os doentes. Sua paciência para com os que, como eu, caminham com dificuldade na aridez da estatística médica fez possível este estudo.

Ao meu amigo e colega de doutorado, Dr. Luís Felipe Piovesan, a minha gratidão pelo suporte incondicional e parceria absoluta em meus projetos profissionais. Crescemos juntos no meu envelhecer e no seu amadurecimento. 
À minha irmã, Winnie Maria Bastian, mulher perfeccionista, determinada e de invejável padrão laboral, que pra mim segue sendo a menina doce que tive o privilégio de carregar nos braços e assistir crescer. Sua casa foi minha morada em São Paulo.

Ao João Eberhardt Francisco pelo suporte nas minhas estadas em São Paulo.

Aos incansáveis, Guilherme Caldas e Eduardo Miranda, que muito contribuíram para o levantamento dos dados deste estudo. Minha profunda gratidão e meu desejo de sucesso em suas carreiras acadêmicas.

À Sra. Elisa de Arruda Cruz da Silva, secretária da pós-graduação do HCFMUSP, por todo o apoio prestado ao longo da trajetória desse doutorado. Pessoa dedicada ao seu trabalho e sempre pronta para, gentilmente, tornar a vida dos alunos mais simples.

Às Sras. Camila Marin, Sanarelly Pires Adonias, Poliana Machado e Astrid Meira, pelo grande apoio à pesquisa que oferecem aos alunos de pós-graduação da Urologia. Seu trabalho na integração dos dados do HCFMUSP com os dados do ICESP foi fundamental para este estudo.

À minha esposa, Luciana, e às minhas filhas, Júlia e Flávia, que todos os dias lapidam minhas asperezas e fazem de mim um sujeito um pouco menos egoísta. Mais da metade dos meus dias estão preenchidos pela presença da Luciana. Sua cumplicidade me estimula sonhar e inspira as minhas realizações. A paternidade é a mais fascinante das jornadas terrenas.

Aos meus pais, que me deram tudo e iniciaram minha caminhada. Com eles aprendi as noções mais fundamentais de liberdade e respeito. 
Esta tese foi escrita e compilada em $\mathrm{HT}_{\mathrm{E}} \mathrm{X}$ e está de acordo com as seguintes normas, em vigor no momento desta publicação:

Referências: adaptado de International Committee of Medical Journals Editors (Vancouver)

Universidade de São Paulo. Faculdade de Medicina. Serviço de Biblioteca e Documentação. Guia de apresentação de dissertações, teses e monografias. Elaborado por Anneliese Carneiro da Cunha, Maria Júlia de A. L. Freddi, Maria F. Crestana, Marinalva de Souza Aragão, Suely Campos Cardoso, Valéria Vilhena. $2^{a}$ ed. São Paulo: Serviço de Biblioteca e Documentação; 2005.

Abreviaturas dos títulos dos periódicos de acordo com List of Journals Indexed in Index Medicus. 


\section{Sumário}

\section{Lista de tabelas}

\section{Lista de figuras}

\section{Lista de símbolos e abreviações}

\section{Resumo}

\section{Summary}

$\begin{array}{ll}\text { Introdução } & \text { p. } 1\end{array}$

1.1 Aspectos históricos . . . . . . . . . . . . p. 1

1.2 Aspectos epidemiológicos . . . . . . . . . . p. 1

1.3 Aspectos diagnósticos e prognósticos . . . . . . . . . p. 3

1.4 Aspectos relacionados à decisão de tratamento . . . . . . . . p . 5

1.5 O papel dos nomogramas . . . . . . . . . . . . p. 6

1.5.1 Sobre o nomograma em validação neste estudo $\ldots \ldots$ p. . . 8

1.5.2 Construção de um nomograma . . . . . . . . . p. 9

1.5.3 Validação estatística de um nomograma . . . . . . . . p.9

1.5.4 Atualização de um nomograma . . . . . . . . . p. 10

$\begin{array}{ll}\text { Objetivos } & \text { p. } 12\end{array}$

$\begin{array}{ll}\text { Método p. } 13 & \text { p }\end{array}$

3.1 Desenho do estudo . . . . . . . . . . . . p. 13

3.2 Local e época do estudo . . . . . . . . . . . . . . p. 13

3.3 População do estudo . . . . . . . . . . . . . . . . p. 14

3.3.1 Critérios de inclusão e exclusão . . . . . . . . . . . . p. 14

3.4 Intervenção $\ldots \ldots \ldots \ldots \ldots \ldots$ p. 15 
3.4.1 Estudo anatomopatológico da biópsia de próstata . . . . . p. 15

3.4 .2 Procedimento cirúrgico . . . . . . . . . . p. 15

3.4.3 Estudo anatomopatológico dos espécimes cirúrgicos . . . . p. 16

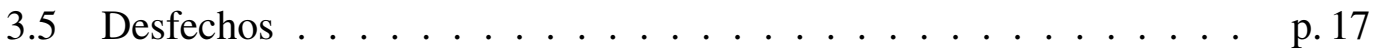

3.6 Análise estatística . . . . . . . . . . . . . . p. 17

3.6.1 Procedimentos para validação externa dos nomogramas de Crippa e Srougi . . . . . . . . . . . . p. 18

3.6.2 Procedimentos para construção dos nomogramas da USP . . p. 19

$\begin{array}{ll}\text { Resultados } & \text { p. } 21\end{array}$

4.1 Validação dos nomogramas de Crippa e Srougi . . . . . . . . p. 21

4.2 Construção dos nomogramas da USP . . . . . . . . . . . . . p. 25

$\begin{array}{ll}\text { Discussão } & \text { p. } 33\end{array}$

$\begin{array}{ll}\text { Conclusões } & \text { p. } 43\end{array}$

$\begin{array}{lr}\text { Anexo A } & \text { p. } 44\end{array}$

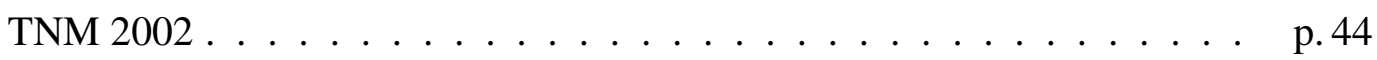

Avaliação do Tumor Primário $(\mathrm{T}) \ldots \ldots$. . . . . . . . . . . . p.44

Avaliação dos Linfonodos . . . . . . . . . . . . . p.44

$\begin{array}{lr}\text { Anexo B } & \text { p. } 45\end{array}$

B.1 Nomogramas para câncer de próstata - revisão 2010 . . . . . . . p. 45

$\begin{array}{lr}\text { Anexo C } & \text { p. } 48\end{array}$

C.1 Nomograma 1 de Crippa e Srougi . . . . . . . . . . . p. 48

C.2 Nomograma 2 de Crippa e Srougi . . . . . . . . . . . . . . p.49

$\begin{array}{lr}\text { Referências } & \text { p. } 50\end{array}$ 


\section{Lista de Tabelas}

1 Características Demográficas . . . . . . . . . . . p. 22

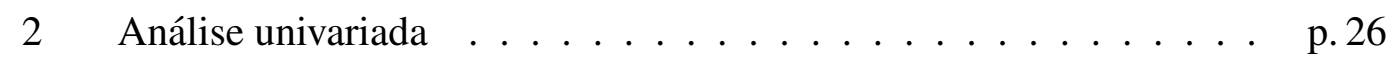

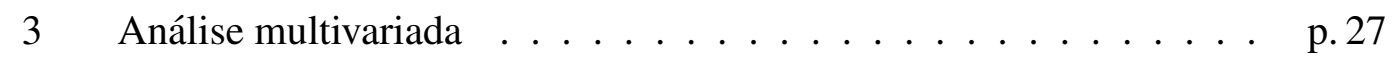

4 Modelos finais de regressão logística . . . . . . . . . . p. 28

5 Nomograma USP: Predição de DOC . . . . . . . . . . . . . p. 29

6 Nomograma USP: Predição de IVS . . . . . . . . . . . . . . . p. 29 


\section{Lista de Figuras}

1 Curva ROC - nomograma 1 de Crippa e Srougi . . . . . . . . p. 23

2 Curva ROC - nomograma 2 de Crippa e Srougi . . . . . . . . p. 23

3 Gráfico de calibração - nomograma 1 de Crippa e Srougi - predição de DOC . . . . . . . . . . . . . . . . . p. 24

4 Gráfico de calibração - nomograma 2 de Crippa e Srougi - predição

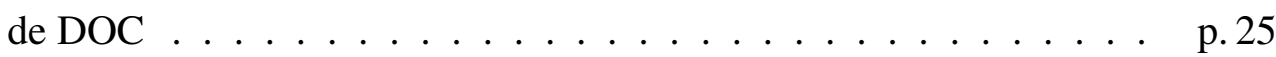

5 Curva ROC - nomograma da USP - predição de DOC - 1.000 amos$\operatorname{tras}$ bootstrap $\ldots \ldots \ldots \ldots \ldots \ldots \ldots \ldots \ldots \ldots$

6 Curva ROC - nomograma da USP - predição de IVS - 1.000 amos-

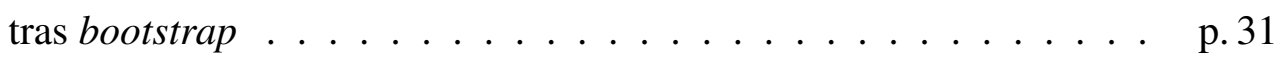

7 Gráfico de calibração - nomograma da USP - predição de DOC 1.000 amostras bootstrap $\ldots \ldots \ldots \ldots$. . . . . . . . . . . . . . .

8 Gráfico de calibração - nomograma da USP - predição de IVS 1.000 amostras bootstrap $\ldots \ldots \ldots$. . . . . . . . . . . . . . . . . 


\section{Lista de Símbolos e Abreviações}

PSA: Antígeno Prostático Específico

PCPT: Prostate Cancer Prevention Trial

MSKCC: Memorial Sloan-Kettering Cancer Center

PFPB: Porcentagem de Fragmentos Positivos na Biópsia de Próstata

DOC: Doença Órgão-Confinada

IVS: Invasão de Vesículas Seminais

OV: Observação Vigilante

HCFMUSP: Hospital das Clínicas da Faculdade de Medicina da Universidade de São Paulo

ICESP: Instituto do Câncer do Estado de São Paulo

EEC: Extensão Extra-Capsular

ROC: Curva de Características Operacionais

ASC: Área Sob a Curva ROC

LOESS: Distribuição atenuada por regressão local não paramétrica 


\section{Resumo}

Bastian Junior AJ. Validação externa de nomograma brasileiro para predição de câncer de próstata órgão-confinado em instituição terciária de ensino. Nomogramas da USP [tese]. São Paulo: Faculdade de Medicina, Universidade de São Paulo; 2011.

Objetivos: (a) Validar externamente os nomogramas de Crippa e Srougi que combinam antígeno prostático específico (PSA) sérico, porcentagem de fragmentos positivos na biópsia prostática (PFPB) e escore de Gleason da biópsia prostática para predição de doença órgão-confinada (DOC), em prostatectomias radicais realizadas entre 1988 e 2002, em uma população contemporânea de instituição de ensino terciária de assistência pública; (b) Re-derivar as variáveis e ajustá-las para produzir dois nomogramas de predição para DOC e invasão de vesículas seminais (IVS), resultando nos nomogramas da USP.

Método: A acurácia (discriminação e calibração) dos nomogramas de Crippa e Srougi foi examinada em 1.002 pacientes submetidos a prostatectomia radical retropúbica (PRR) entre 2005 e 2010 na Universidade de São Paulo. Curvas ROC com as respectivas áreas sob a curva (ASC) e escores de Brier foram usados para quantificar as propriedades discriminativas das predições dos nomogramas de Crippa e Srougi para DOC. Gráficos LOESS de calibração foram usados para expressar visualmente a relação entre as probabilidades preditas e as taxas de desfecho observadas. Na sequência, rederivação das variáveis incluindo PSA, PFPB, escore de Gleason da biópsia prostática e estadio clínico foi realizada para os desfechos DOC e IVS. Modelos finais de regressão logística originaram os nomogramas de predição para DOC e IVS (intervalos de confiança de $95 \%$ em 1.000 amostras bootstrap). Os procedimentos de validação acima descritos foram aplicados na validação interna dos nomogramas da USP.

Resultados: Os nomogramas de Crippa e Srougi para predição de DOC apresentaram ROC ASC=0,68 (IC: 0,65-0,70), escore de Brier de 0,17 e considerável subestimação nos gráficos LOESS. Os nomogramas da USP apresentaram ROC ASC $=0,73$ (IC: 0,700,76) e escore de Brier de 0,16 para DOC e ROC ASC=0,77 (IC: 0,73-0,79) e escore de Brier de 0,08 para IVS. Os gráficos LOESS mostraram excelente calibração para DOC e considerável superestimação de desfecho para IVS em todas as faixas de predição acima de $2 \%$.

Conclusões: Os nomogramas de Crippa e Srougi foram validados externamente apresentando discriminação moderada e considerável subestimação de desfecho para DOC. Os nomogramas da USP apresentaram boa discriminação para DOC e IVS, excelente calibração para DOC e considerável superestimação de desfecho para IVS.

Descritores: Neoplasias da Próstata, Nomogramas, Prostatectomia, Prognóstico, Modelos Logísticos, Análise de Regressão 


\section{Summary}

Bastian Junior AJ. External validation of a Brazilian predictive nomogram for pathologic outcomes following radical prostatectomy in tertiary teaching institutions: the USP nomograms [thesis]. São Paulo: Faculdade de Medicina, Universidade de São Paulo; 2011.

Purposes: (a) To externally validate the Crippa and Srougi' nomograms that combine serum prostate-specific antigen (PSA), percent of positive biopsy cores (PPBC), and biopsy Gleason score to provide the likelihood of organ-confined disease (OCD) in radical prostatectomy performed between 1988 and 2002 in a contemporary cohort of a public health environment of a teaching institution; (b) To re-derivate the variables and adjust them to produce two predictive nomograms for OCD and seminal vesicle invasion (SVI), the USP nomograms.

Material and Methods: The discrimination and calibration properties of the nomograms from Crippa and Srougi in 1002 men who underwent radical prostatectomy between 2005 and 2010 at the University of São Paulo (USP) were examined. The ROC derived area under the curve (AUC) and the Brier score were used to quantify the discriminant properties of the predictions of that nomograms for OCD. LOESS based calibration plots were used to examine the relationship between the predicted and observed rates of OCD. Furthermore, variables re-derivation including PSA, PPBC, biopsy Gleason score and clinical stage was done for OCD and SVI outcomes. Logistic regression final models resulted in predictive nomograms for OCD and SVI (1000 bootstrap 95\% confidence intervals). The abovementioned validation procedures were applied in USP nomograms internal validation.

Results: The OCD prediction nomograms from Crippa and Srougi showed ROC AUC $=0.68$ (CI:0.65-0.70) and Brier score of 0.17; and considerable underestimation in LOESS plots. The USP nomograms showed ROC AUC $=0.73$ (CI:0.70-0.76) and Brier score of 0,16 for OCD and ROC AUC $=0.77$ (CI: 0.73-0.79) and Brier score of 0,08 for SVI. The LOESS plots showed excellent calibration for OCD and overestimation for SVI in all prediction ranges over $2 \%$.

Conclusions: The nomograms from Crippa and Srougi were externally validated showing moderate discrimination and considerable OCD underestimation. The USP nomograms showed good discrimination for both, OCD and SVI, excellent calibration for OCD and considerable overestimation for SVI.

Descriptors: Prostatic Neoplasms, Nomograms, Prostatectomy, Prognosis, Logistic Models, Regression Analysis 


\section{Introdução}

\subsection{Aspectos históricos}

Os primórdios da descrição do câncer de próstata remontam aproximadamente $200 \operatorname{anos}^{1}$. O primeiro caso de câncer de próstata confirmado por estudo histológico foi descrito em 1853 por Adams, em Londres, no Royal Medical and Chirurgical Society of London e considerado como uma doença muito rara ${ }^{2}$. Apesar de haver relatos anteriores descrevendo poucos casos de abordagem perineal e/ou abdominal de massas tumorais prostáticas obstrutivas, Young em 1905 descreveu os primeiros quatro casos de prostatectomia radical perineal ${ }^{3}$. Em 1947, Millin introduziu a prostatectomia radical retropúbica ${ }^{4}$ e, em 1983, Walsh modificou sua técnica ligando o complexo da veia dorsal do pênis, identificando e preservando os feixes vasculonervosos relacionados à preservação da função erétil ${ }^{5}$.

\subsection{Aspectos epidemiológicos}

A prevalência do câncer de próstata é elevada nos homens acima dos 45 anos. Sua progressão tem relação direta com a idade, sendo histologicamente evidente em 46 a $70 \%$ dos homens acima dos $70 \operatorname{anos}^{6-8}$.

O câncer de próstata tem sido a neoplasia maligna visceral mais comum em homens nos EUA desde 1984, agora responsável por $28 \%$ do total de tais neoplasias, sendo este a segunda causa de morte por câncer no sexo masculino naquele país. O 
risco estimado da doença ao longo da vida é de $15,39 \%$ para os homens brancos e de $18,32 \%$ para os afro-americanos., 9

Com relação à condição socioeconômica das pessoas, a taxa de mortalidade por câncer de próstata é 1,5 vezes maior nos homens brancos com até 12 anos de escolaridade e 2 vezes maior nos homens afrodescendentes com até 12 anos de escolaridade, quando comparados com os mesmos grupos raciais com mais de 12 anos de escolaridade. Esse aspecto é relevante porque apresenta como primeira hipótese a diferença de acesso ao diagnóstico precoce através do Antígeno Prostático Específico (PSA) ${ }^{11,12}$.

No cenário mundial, a incidência do câncer de próstata varia grandemente entre os países e populações étnicas, chegando a diferenças da grandeza de cem vezes. A menor incidência anual ocorre na Ásia e a maior ocorre na Escandinávia e na América do Norte, especialmente entre os afro-americanos. A mortalidade também varia amplamente entre as nações, sendo mais alta na Suécia e mais baixa na Ásia ${ }^{13}$.

Dentre os países em desenvolvimento destacaram-se em termos de incidência o Caribe, os países da América do Sul e da África subsaariana. Com relação à mortalidade, o câncer de próstata apresentou-se como a sexta causa de morte por câncer no sexo masculino na população mundial, totalizando 258.000 óbitos em $2008^{14}$.

No Brasil, as estimativas para o ano de 2010 apontam que ocorrerão 489.270 casos novos de câncer (236.240 casos novos para o sexo masculino e 253.030 para sexo feminino). Segundo estimativa do Instituto Nacional do Câncer e do Ministério da Saúde, o coeficiente bruto de mortalidade por câncer de próstata no Brasil aumentou, entre 1979 e 2007 , de 3,73 por 100.000 homens para 12,34 por 100.000 homens $^{15}$.

A distribuição dos casos novos de câncer segundo localização primária é bem heterogênea entre estados e capitais do país. O câncer de próstata é o mais frequente na região centro-oeste, com risco estimado de 44 por 100.000 homens, e o segundo em todas as demais regiões depois dos tumores de pele não-melanoma, com risco estimado 
de 69 por 100.000 homens na região Sul, 62 por 100.000 homens na região Sudeste, 44 por 100.000 homens na região Nordeste e 24 por 100.000 homens na região Norte. No estado de São Paulo a estimativa de casos novos de câncer de próstata para 2010 é de 13.160 novos casos, com taxa bruta de incidência de 61,84 por 100.000 homens; e na cidade de São Paulo estima-se 4.110 novos casos, com respectiva taxa bruta de incidência de 70,48 por 100.000 homens ${ }^{16}$.

\subsection{Aspectos diagnósticos e prognósticos}

O PSA é abundante no epitélio prostático e é virtualmente órgão-específico. Como é expressado tanto no tecido prostático normal como na glândula hiperplásica e na neoplasia de próstata, apresenta pobre especificidade na discriminação entre condições patológicas benignas e câncer de próstata, especialmente hiperplasia prostática benigna que resulta em aumento do PSA e da calicreína humana 2 (hK2). A especificidade do PSA é mais problemática no intervalo de resultados entre 4 e 10 a $15 \mathrm{ng} / \mathrm{dl}$, resultando em índices de biópsias negativas de 70 a $80 \%{ }^{17,18}$. A despeito de vários pontos de corte propostos para o PSA total, falta um valor capaz de separar homens com alto risco de câncer de próstata daqueles com baixo risco de apresentarem a doença, ou de separar homens com câncer de próstata clinicamente significativo daqueles com neoplasia indolente. Essa questão foi claramente ilustrada nas análises realizadas com os dados do braço placebo do Prostate Cancer Prevention Trial (PCPT) ${ }^{19}$, um grande estudo clínico randomizado sobre finasterida para prevenção do câncer de próstata. Sugere-se que o ponto de corte do PSA de 4 ng/dl é arbitrário e que baixar o parâmetro de referência do PSA pode ser benéfico para identificar homens com considerável risco de desenvolverem câncer de próstata a longo $\operatorname{prazo}^{19,20}$.

São fatores prognósticos reconhecidamente independentes o estadio clínico (determinado pelo toque retal) e patológico, o escore de Gleason ${ }^{21}$ e o PSA, os quais ajudam 
a estratificar os pacientes em grupos de risco, muito úteis para o estadiamento e conduta terapêutica. Também a micro-invasão vascular ${ }^{22}$ guarda relação com o prognóstico do câncer de próstata. No intuito de subestratificar os grupos de risco e individualizar condutas, tem-se utilizado ferramentas preditoras como as Tabela de Partin e os nomogramas de Kattan (MSKCC), que podem ajudar a prever o estadio patológico e o risco de recidiva após o tratamento ${ }^{23,24}$.

A porcentagem de fragmentos positivos na biópsia de próstata (PFPB) é um dado importante para prever a doença órgão-confinada (DOC). Conforme demonstrado por D’Amico, 79\% dos paciente que apresentam menos de 34\% dos fragmentos da biópsia acometidos por neoplasia possuem doença restrita à glândula, enquanto essa condição existe em apenas $43 \%$ dos pacientes que possuem mais de $50 \%$ dos fragmentos positivos. O mesmo estudo demonstra que nos pacientes com menos de $34 \%$ de fragmentos positivos apenas $11 \%$ apresentaram recidiva da doença após a prostatectomia radical, enquanto esse evento ocorreu em $37 \%$ dos pacientes com mais de $50 \%$ de fragmentos $\operatorname{positivos}^{25}$.

Também o acometimento das vesículas seminais na peça cirúrgica guarda relação com o número de fragmentos positivos encontrados na biópsia da próstata, conforme evidenciaram Peller et al., demonstrando que, quando apenas um fragmento da biópsia é positivo, a chance de comprometimento de vesículas seminais é de apenas $6 \%$, ao passo que essa chance salta para $83 \%$ quando há 6 fragmentos da biópsia positivos ${ }^{26}$.

D’Amico estratifica os pacientes em três grupos de risco (Baixo, Intermediário e Alto), de acordo com os dados de PSA pré-tratamento, estadiamento clínico pelo toque retal e escore de Gleason dos fragmentos da biópsia. Em tese, os valores de PSA se correlacionam com o volume tumoral, as características do tumor ao toque retal com a extensão da doença e o escore de Gleason do tumor com sua agressividade ${ }^{27}$. 


\subsection{Aspectos relacionados à decisão de tratamento}

O célebre dilema acerca do tratamento do câncer de próstata levantado pelo Dr. Willet Whitmore continua atual e desafiador: A cura é necessária quando possível? E a cura é possível quando necessária?

Segundo Touijer e Scardino, estratificar os pacientes com câncer de próstata clinicamente localizado em grupos cuja base é a existência de características prognósticas comuns tem sido a forma mais utilizada para atender à complexidade do dilema do Dr. Whitmore, sendo a classificação por grupos de risco de D'Amico a mais empregada e que demonstra uma correlação prognóstica com cada um dos grupos. Apesar de muito útil no dia a dia e de fácil realização, essa estratificação é simplista e sujeita a erros na medida em que a própria realidade epidemiológica do câncer da próstata pode mudar com o tempo, fazendo com que os casos sejam diagnosticados mais precocemente e, portanto, diminuindo o peso do estadiamento clínico no processo decisório ${ }^{28}$.

Ainda, segundo os mesmos autores, o complexo cenário do diagnóstico do câncer de próstata, bem como do processo decisório sobre as formas de tratamento, estabelece quatro possibilidades no momento de orientar o tratamento do doente: (a) negar a habilidade de prever o desfecho da doença no nível individual do paciente; (b) tentar prever o desfecho baseado no julgamento clínico e na experiência do especialista; (c) tentar prever o desfecho estratificando por grupos de risco; e (d) acessar informações específicas como o valor do PSA, o estadio clínico da doença, o escore de Gleason na biópsia, a proporção de comprometimento de cada fragmento na biópsia, a PFPB, o estado geral do paciente e a idade e utilizar ferramentas de predição como nomogramas ou algoritmos.

O estadiamento do CaP é crítico para o sucesso do tratamento curativo dessa neoplasia, onde a presença ou não de extensão extraprostática e a condição de invasão das vesículas seminais (IVS) influenciam a escolha do tratamento, as chances de cura 
e, no caso do tratamento cirúrgico, a decisão a respeito da estratégia de preservação dos feixes vasculonervosos responsáveis pela função erétil ${ }^{29}$. Junto com a crescente proporção de homens que apresentam tumor impalpável (T1c) ao diagnóstico, cresce também o número de pacientes que são conduzidos com observação vigilante (OV) e os critérios para tal conduta são controversos e cruciais para a sua correta condução ${ }^{30}$.

\subsection{O papel dos nomogramas}

Na medida em que mais variáveis relacionadas com a extensão da doença ou com os parâmetros de resultado após o tratamento se tornam disponíveis para o uso clínico, mais complexo fica o processo de decisão. Um grande número de publicações descrevendo ferramentas de auxílio ao diagnóstico e à tomada de decisão vem aparecendo na literatura médica especializada nas últimas duas décadas.

Esses modelos de auxílio ao diagnóstico e à tomada de decisão são considerados de predição quando avaliam a probabilidade de um determinado desfecho sem considerar o efeito do tempo, e de prognóstico quando avaliam a probabilidade de um determinado desfecho ao longo de um período de tempo definido.

Numa recente revisão sistemática da literatura sobre esse tema aplicado especificamente ao câncer de próstata clinicamente localizado, Lughezzani et al. selecionaram 16 modelos de predição e 22 modelos de prognóstico cujo desenho do estudo modelo e da validação, interna ou externa, consideraram adequados para originar novas validações ou justificar seu uso clínico ${ }^{31}$. O citado autor classificou os nomogramas existentes para câncer de próstata seguindo a estratificação por características funcionais, dividindo-os em (a) Nomogramas preditivos: Aumento de escore de Gleason na peça cirúrgica, predição de estadio patológico e predição de desfechos funcionais (função erétil e continência urinária); e (b) Nomogramas prognósticos: Mortalidade, mortalidade câncer-específica e expectativa de vida. O Anexo B.1 reproduz as tabelas 
do autor ilustrando de forma muito objetiva a atual realidade dos modelos de predição existentes na literatura, que já progrediram nas fases básicas da hierarquia de evidência para uso clínico.

Ainda, segundo Lughezzani et al. ${ }^{31}$, são as seguintes as principais limitações de um nomograma de predição ou de prognóstico para uso em câncer de próstata: (a) falta de validação externa; (b) falta de atualização periódica - As mudança nas características da doença, dos métodos diagnósticos ou da população ao longo do tempo podem afetar consideravelmente a performance de um nomograma; (c) falta de variáveis novas; (d) falta de flexibilidade; e (e) incapacidade de quantificar o benefício da prostatectomia radical em comparação com os resultados das outras formas de tratamento existentes para o câncer de próstata clinicamente localizado.

A hierarquia apresentada por McGinn et al. ${ }^{32}$ em seu guia para uso de modelos de decisão clínica estabelece 4 níveis de evidência para os modelos de predição, a saber:

- Nível 4: Nomogramas que necessitam melhor avaliação antes de serem usados na prática clínica. Aqui estão classificados os modelos que foram apenas derivados e não validados, apresentam apenas validação interna ou validação meramente estatística.

- Nível 3: Nomogramas que podem ser usados clinicamente com muito critério desde que a população em que serão empregados seja muito similar do ponto de vista demográfico e estrutural com relação à doença em questão. Referem-se aos estudos validados prospectivamente em apenas uma amostra de pequeno número de pacientes.

- Nível 2: Nomogramas que podem ser usados em vários contextos com confiança em sua acurácia. São modelos que demonstraram acurácia, validados externamente em pelo menos uma amostra de grande número de pacientes ou validados externamente em várias pequenas amostras estruturalmente muito distintas da população do estudo modelo.

- Nível 1: Nomogramas que podem ser usados em uma grande variedade de contextos com confiança de que podem produzir mudanças de comportamento clínico e/ou melhorar o desfecho dos pacientes. 


\subsubsection{Sobre o nomograma em validação neste estudo}

Em 2004, Crippa e Srougi, com o propósito de criação de um nomograma com o contexto de pacientes latino-americanos, desenvolveram um nomograma de predição de DOC com base em três variáveis: PSA total, escore de Gleason na biópsia de próstata e $\mathrm{PFPB}^{33}$. O estudo foi realizado de maneira retrospectiva, avaliando uma casuística de 898 pacientes com câncer de próstata clinicamente localizado submetidos a prostatectomia radical retropúbica pelo mesmo cirurgião. O processo de derivação das variáveis do modelo foi obtido por meio de regressão logística das possíveis variáveis preditoras, inicialmente compostas pelo valor total do PSA, estadio clínico baseado no toque retal de acordo com a classificação de TNM de $1992^{34}$, escore de Gleason do carcinoma prostático na biópsia e PFPB. A análise de regressão logística elegeu como variáveis finais do modelo o PSA total, o escore de Gleason da biópsia e a PFPB. A análise de regressão logística multivariada baseada nessas três variáveis resultou em dois submodelos, o primeiro deles agrupando os valores de PSA em quatro classes $(0-4 ; 4,1-10 ; 10,1-20 ;$ e $>10)$, agrupando os escores de Gleason em três classes (2-6; 7 e 9-10) e agrupando a PFPB em quatro classes $(0-25 \% ; 25,1-50 \% ; 50,1-75 \%$ e 75-100\%). O segundo foi criado agrupando as classes da variável escore de Gleason da biópsia em 2-6 e 7-10. A comparação entre os dois submodelos não mostrou diferença estatisticamente significativa, o que fez com que os autores sugerissem o segundo submodelo em virtude da maior simplicidade de uso. A validação interna dos dois submodelos foi realizada pelo método de bootstrapping ${ }^{35}$, que resultou em correta predição do desfecho (estadio patológico) em respectivamente $87 \%$ e $91 \%$ dos casos. A excelente performance de predição de DOC apresentada pelo nomograma, confirmada pela validação interna realizada foi publicada em 2006, porém, encontra-se até o momento na condição de nível de evidência 4, haja vista a pendência de validação externa em população diferente da população de origem. 


\subsubsection{Construção de um nomograma}

A construção de um nomograma de predição passa necessariamente por duas fases: a derivação das variáveis e construção do modelo por regressão logística das variáveis preditoras que melhor performance apresentaram na regressão e a validação interna da performance preditiva do nomograma realizada na mesma população do estudo modelo. Essa validação interna pode ser realizada no mesmo momento da criação do modelo, usando uma parte da população do estudo reservada para o processo de validação ou por meio de seleção de pequenos grupos de indivíduos aleatoriamente retirados da população estudada e submetidos ao nomograma. Esse processo repetido sucessivas vezes dentro da mesma amostra recebe a denominação de bootstrapping e é um método bastante utilizado como validação interna nos nomogramas de predição ${ }^{35}$. Quando a validação interna do modelo de predição é realizada posteriormente ao estudo, de forma prospectiva com pacientes originados da mesma população que originou o modelo, é denominada validação temporal. Vale ressaltar que essas formas de validação apresentam considerável possibilidade de erro randômico e portanto estabelecem, para qualquer forma de modelo de predição, a necessidade implícita de estudo de validação externa do modelo para que a possibilidade de uso clínico do modelo de predição possa ser cogitada.

\subsubsection{Validação estatística de um nomograma}

A validação externa pode ser geográfica ou de domínio. Por validação geográfica entende-se a aplicação do modelo de predição em estudo a uma população geograficamente distinta, mas que guarde similaridades com a população original do modelo em termos de incidência do desfecho e definição das variáveis. A validação de domínio existe quando o contexto da instituição em que o nomograma do estudo modelo será 
aplicado é consideravelmente diverso do contexto que originou o modelo, seja em termos de diferenças populacionais, diferenças de incidência de desfecho ou diferenças de conceituação, coleta ou aferição das variáveis. Tanto a validação geográfica quanto a validação de domínio podem ser realizadas de forma prospectiva ou retrospectiva.

O objetivo mais importante no processo de validação de um nomograma é estabelecer, comparativamente com o estudo modelo, a performance de predição do mesmo. Os aspectos a serem avaliados são: discriminação, calibração, generalização (ou transportabilidade), nível de complexidade e benefício clínico. Desses quesitos, os mais importantes são o poder de discriminação e a calibração. Por discriminação entende-se a probabilidade de o modelo predizer corretamente o desfecho para o paciente quando sorteado entre os demais. Por calibração entende-se a relação entre as probabilidades preditas e as taxas observadas do desfecho, incluindo todas as instâncias do modelo de predição em validação. A inclusão dessa análise na validação de modelos de predição é essencial porque o modelo pode predizer muito bem em pacientes com baixo risco para o desfecho, mas ter performance muito pobre nos casos onde o risco de desfecho é maior.

Segundo Justice et al. as ferramentas de predição se mostram mais robustas na medida em que são cumulativamente testadas em ambientes sucessivamente distintos. Quanto mais diversos os contextos de validação externa em que a acurácia (discriminação e calibração) de um modelo é confirmada, maior a probabilidade de ser generalizada para uma população não testada ${ }^{36}$.

\subsubsection{Atualização de um nomograma}

Quando um ou mais estudos de validação externa de um nomograma apresenta resultados desapontadores em termos de performance de predição, a alternativa mais 
óbvia para muitos autores é criar um novo modelo de predição, geralmente desprezando os dados do modelo anterior e trazendo para o contexto científico mais um nomograma para o mesmo desfecho, sem ter esgotado as possibilidades do modelo anterior. Uma alternativa altamente estimulada é a atualização do modelo de predição com os dados da população em que o modelo se mostrou insuficientemente validado, geralmente resultando em adaptação do modelo às características da nova população e aumento de sua transportabilidade ${ }^{37}$.

O Hospital das Clínicas da Faculdade de Medicina da Universidade de São Paulo (HCFMUSP) e o Instituto do Câncer do Estado de São Paulo (ICESP) figuram entre as maiores instituições públicas de referência para o tratamento do $\mathrm{CaP}$ no Brasil. Sendo centros terciários, sua população de pacientes com câncer de próstata é consideravelmente heterogênea no que diz respeito às variáveis preditoras do estadio patológico do CaP. Sendo Centros de ensino, os procedimentos cirúrgicos e de análise patológica das peças cirúrgicas são realizados por médicos residentes, sob supervisão, em diferentes níveis de treinamento.

Tem-se como hipótese que a validação externa em cenário de tal heterogeneidade pode (a) desafiar significativamente as capacidades de generalização e transportabilidade de um nomograma construído em população mais homogênea e, (b) exigir o ajuste das variáveis do nomograma para acomodar as características das populações assistidas em centros terciários públicos e ambientes de ensino.

Neste cenário, a utilização de um nomograma como o de Crippa e Srougi, uma vez validado externamente, pode objetivar e homogeneizar o processo decisório e a dinâmica de informar o paciente sobre sua doença e tratamentos propostos. 


\section{Objetivos}

1. Realizar a validação externa do nomograma desenvolvido por Crippa e Srougi para predição de doença órgão-confinada.

2. Desenvolver nomogramas ajustados para predição de doença órgão-confinada e de invasão de vesículas seminais em pacientes com câncer de próstata, aplicáveis à população assistida pelo HCFMUSP e pelo ICESP. 


\section{Método}

Este estudo foi aprovado pelo Comissão de Ética para Análise de Projetos de Pesquisa do HCFMUSP, com dispensa do consentimento informado.

\subsection{Desenho do estudo}

Trata-se de um estudo retrospectivo, observacional, realizado através da revisão de prontuários médicos clínicos registrados eletronicamente nas instituições onde o estudo foi realizado, de pacientes submetidos a Prostatectomia Radical para tratamento do câncer de próstata.

\subsection{Local e época do estudo}

Pacientes tratados cirurgicamente no Hospital das Clínicas da Faculdade de Medicina da Universidade de São Paulo (HCFMUSP) ou no Instituto do Câncer do Estado de São Paulo (ICESP). As duas instituições atendem à demanda cirúrgica oncológica de pacientes atendidos pela Divisão de Clínica Urológica do HCFMUSP. Ambas são instituições hospitalares de natureza pública, que dão atendimento a pacientes do Sistema Único de Saúde - SUS. O estudo abrangeu pacientes operados entre Janeiro de 2005 e Dezembro de 2010. 


\subsection{População do estudo}

Do total de 1.094 pacientes submetidos a Prostatectomia Radical no período do estudo, foram estudados 1.002 pacientes do sexo masculino com diagnóstico de câncer de próstata clinicamente localizado e submetidos a Prostatectomia Radical Retropúbica Aberta. A idade dos pacientes variou entre 44 e 81 anos, com a idade média de 64,7 e mediana de 65 anos.

Todos os pacientes foram submetidos ao estadiamento clínico da doença com base na classificação TNM de $2002^{38}$ representada no Anexo A. Todos os pacientes incluídos no estudo receberam a classificação de T estabelecida unicamente pelo toque retal, realizado pelo médico residente de urologia e confirmado pelo médico urologista do HCFMUSP/ICESP responsável por seu atendimento, e tiveram seus dados de biópsia registrados. Todos os pacientes tiveram seu resultado de PSA pré-operatório atualizado dentro das instituições do estudo quando o mesmo tinha mais de 90 dias ou era de resultado duvidoso. Em virtude da realidade de referência em tratamento oncológico vivida pelas instituições onde o estudo foi realizado, na quase totalidade dos pacientes do estudo a biópsia de próstata foi realizada em outro serviço, portanto, os dados de biópsia incluídos no estudo foram baseados unicamente nas descrições de laudo das mesmas, independente da instituição de origem. Os dados anatomopatológicos da biópsia de próstata foram registrados de forma sistemática, incluindo o número total de fragmentos obtidos, número de fragmentos positivos e classificação de Gleason ${ }^{21}$, estratificada em componente primário, componente secundário e escore total.

\subsubsection{Critérios de inclusão e exclusão}

Dos 1.094 paciente submetidos a prostatectomia radical nas duas instituições foram selecionados os pacientes que apresentaram os dados de PSA pré-operatório, 
biópsia de próstata sistematicamente descrita e descrição do item $\mathrm{T}$ da classificação de TNM 2002 registrados de forma clara no seu prontuário.

Os critérios de exclusão foram: (a) prontuário incompleto ou com registro conflitante dos dados avaliados no estudo; (b) uso de qualquer forma de tratamento hormonal neoadjuvante e; (c) diagnóstico da neoplasia prostática realizado em fragmentos oriundos de cirurgia endoscópica da próstata.

\subsection{Intervenção}

\subsubsection{Estudo anatomopatológico da biópsia de próstata}

Em virtude da característica assistencial de referência em câncer urológico das duas instituições envolvidas no estudo, os dados anatomopatológicos da biópsia de próstata dos pacientes, em sua imensa maioria, vieram de fora dessas instituições, acompanhando os pacientes em questão e embasando o diagnóstico de câncer de próstata que motivou o encaminhamento.

A descrição da classificação histológica de Gleason ${ }^{21,39}$ foi adotada exatamente como apresentada no laudo anatomopatológico recebido com o paciente, obedecendo a ordem exata dos padrões primário e secundário, sendo o escore o resultado da soma aritmética simples dos dois padrões.

\subsubsection{Procedimento cirúrgico}

Os pacientes foram submetidos a tratamento cirúrgico do câncer de próstata clinicamente localizado através da prostatectomia radical retropúbica descrita por Walsh ${ }^{40}$ e modificada por Srougi ${ }^{41}$. Em virtude das características docentes de ambas as instituições envolvidas no estudo, os pacientes foram operados pelos médicos residentes 
da Divisão de Clínica Urológica do HCFMUSP, sob supervisão direta do médico urologista docente responsável pelo caso, tendo sido a mesma técnica cirúrgica realizada em todos os pacientes.

\subsubsection{Estudo anatomopatológico dos espécimes cirúrgicos}

Todos os espécimes cirúrgicos constando da próstata, vesículas seminais e linfonodos obturadores (quando retirados na intervenção cirúrgica) foram analisados pelo Serviço de Anatomia Patológica do Hospital das Clínicas, tendo seus laudos descritos nos sistemas de prontuário eletrônico das instituições envolvidas no estudo. De forma similar ao realizado com as informações da biópsia de próstata, os dados anatomopatológicos da peça cirúrgica também foram obtidos exclusivamente da descrição do laudo emitido pelo médico patologista responsável. Os exames foram realizados por médicos patologistas diferentes, porém, obedecendo as mesmas rotinas de padronização do método existentes na instituição.

A classificação histológica de Gleason foi utilizada para a descrição da diferenciação tumoral, levando em conta o padrão acinar da neoplasia estudada. A utilização dos cinco padrões tumorais reconhecidos bem como o escore obedeceram a reconhecida Classificação de Gleason ${ }^{21,39}$

Como infiltração do tecido periprostático, caracterizando doença não órgão-confinada, considerou-se a invasão do tecido adiposo e/ou do feixe vasculonervoso periprostático.

Como infiltração das vesículas seminais considerou-se a sua invasão pela neoplasia claramente identificada através de invasão por contiguidade em qualquer de suas regiões, desde que não limitada apenas à sua região adventicial. 
Como metástases linfonodais considerou-se o envolvimento dos linfonodos obturadores (quando retirados durante o procedimento cirúrgico), em qualquer número, com qualquer extensão.

O sistema de estadiamento anatomopatológico adotado no estudo foi o sistema TNM de estadiamento ${ }^{38}$.

\subsection{Desfechos}

Para o presente estudo 2 categorias de desfecho binário de interesse foram definidas: 1- doença órgão-confinada (DOC); e 2- invasão de vesículas seminais (IVS). Essas categorias foram mutuamente excludentes, tendo sido considerado o desfecho de maior gravidade. Como o estudo original de Crippa e Srougi apresenta os nomogramas de predição apenas para DOC, a validação daquele estudo com a presente casuística foi limitada a esse desfecho. Para construção dos nomogramas da USP foram considerados DOC e IVS.

\subsection{Análise estatística}

Os critérios de Peduzzi et al. ${ }^{42}$ foram utilizados para estimar o tamanho da amostra do presente estudo. Com base no estudo de Crippa e Srougi ${ }^{33}$, a proporção de DOC foi de $0,34(\mathrm{p})$. Como quatro variáveis $(\mathrm{k})$ foram estudadas no modelo original daquele autor, tamanhos de amostra (n) foram estimados para um mínimo de 10 e um máximo de 25 eventos por variável (EPV) através da fórmula $n=(E P V . k) / p$, resultando em 118 e 294 casos, respectivamente. Como a presente casuística contém registros de 1.002 pacientes, foi considerada adequada para o estudo.

Este estudo foi baseado na premissa de que a presente população se apresentaria de forma significativamente diferente da população do estudo de Crippa e Srougi. Para 
testar essa hipótese, os dados demográficos, incluindo estadio clínico, valores de PSA e dados anatomopatológicos das biópsias prostáticas e das peças cirúrgicas apresentados na tabela 1 do estudo original foram comparados com os dados da população do presente estudo por testes t e testes z para médias e desvios-padrão e proporções conforme apropriado. Todos os testes estatísticos foram bicaudais.

\subsubsection{Procedimentos para validação externa dos nomogramas de Crippa e Srougi}

Os nomogramas de Crippa e Srougi foram desenhados para predição de doença órgão-confinada.

As probabilidades de DOC foram atribuídas aos 1.002 casos do presente estudo de acordo com as tabelas de probabilidade de DOC publicadas pelo autor ${ }^{33}$, apresentadas nos Anexos C.1 e C.2. Aos pacientes de cada grupo de estratificação, levando em conta o PSA (0-4 ng/ml; 4,1-10 ng/ml; 10,1-20 ng/ml e >20 ng/ml ), o escore de Gleason (2-6; 7 e 8-10 no nomograma 1; e 2-6 e 7-10 no nomograma 2) e PFPB (0-25\%; 25,1-50\%; 50,1-75\% e 75,1-100\%) foram atribuídas as probabilidades médias de cada grupo de estratificação dos respectivos nomogramas daquele autor. Essas probabilidades atribuídas foram utilizadas nos testes de validação a seguir descritos.

Curvas de características operacionais (ROC) confrontando as probabilidades atribuídas com as taxas de desfecho foram construídas para acessar a capacidade dos nomogramas de Crippa e Srougi em discriminar os pacientes com relação a DOC, e a área sob a curva (ASC) foi calculada balizada pelo conceito de que a $A S C=1$ representa discriminação perfeita e $A S C=0,5$ representa nenhuma discriminação.

Para representar quantitativamente o desempenho geral dos nomogramas de Crippa e Srougi foram calculados os escores de Brier para cada um dos nomogramas. O escore de Brier representa o desvio quadrado médio entre as probabilidades atribuídas 
para o desfecho (DOC) e as taxas reais observadas na população estudada. O escore igual a 0 indica discriminação perfeita e 0,25 ausência total de capacidade discriminativa do modelo preditivo.

Para explorar graficamente as condições de super ou subestimação do desfecho e aferir a calibração dos nomogramas em validação, a relação entre as probabilidades atribuídas e as taxas observadas de DOC foram representadas por curvas de distribuição atenuadas por regressão local não paramétrica (LOESS) ${ }^{43}$. A coincidência da curva com a linha diagonal indica boa calibração do modelo ao longo das faixas de predição.

\subsubsection{Procedimentos para construção dos nomogramas da USP}

Os nomogramas da USP foram construídos objetivando prever dois desfechos: doença órgão-confinada e invasão de vesículas seminais. As variáveis disponíveis e incluídas no processo de derivação foram PSA, estadio clínico, escore de Gleason da biópsia prostática e porcentagem de fragmentos positivos na biópsia prostática.

A PFPB foi inicialmente dividida em quatro categorias: $0-25 \% ; 25,1-50 \%$; 50,1$75 \%$ e $75,1-100 \%$. Numa segunda análise as categorias $25,1-50 \%$ e $50,1-75 \%$ foram agrupadas. O valor do PSA foi inicialmente dividido em quatro categorias: 0-4 ng/ml; 4,1-10 ng/ml; 10,1-20 ng/ml e $>20 \mathrm{ng} / \mathrm{ml}$. Numa segunda análise as categorias 0-4 ng/dl e 4,1-10 ng/dl foram agrupadas. O escore de Gleason das biópsias de próstata foi dividido, numa primeira análise, em três categorias: 2-6; 7 e 8-10. Numa segunda análise o escore de Gleason foi dividido em duas categorias: 2-6 e 7-10. O colapso de categorias acima descrito foi realizado para aumentar a representatividade nos grupos extremos dos nomogramas de predição sem prejuízo da significância das categorias nos modelos finais de regressão. 
A associação univariada entre as variáveis preditoras e os desfechos binários em questão foi estudada através de análise do qui-quadrado de Pearson.

As variáveis significativamente associadas com cada um dos desfechos foram então submetidas à análise de regressão logística para identificar os preditores independentes para os respectivos desfechos. Os coeficientes e as razões de chance com os respectivos intervalos de confiança de $95 \%$ foram definidos em 1.000 amostras obtidas da coorte original pelo método de bootstrap ${ }^{35}$. Testes de Hosmer \& Lemeshow foram utilizados para assegurar a adequação dos modelos. As curvas ROC com as respectivas áreas sob a curva, os escores de Brier e as curvas de distribuição atenuadas por regressão local não paramétrica (LOESS) ${ }^{43}$ foram desenvolvidos como descrito na seção 3.6.1.

As probabilidades obtidas pelos modelos finais de regressão logística levaram à construção dos nomogramas da USP para previsão de DOC e IVS, e o intervalo de confiança de $95 \%$ foi definido mediante a repetição da análise em 1.000 amostras de bootstrap obtidas a partir da coorte original ${ }^{35}$.

O nível de significância de $5 \%$ foi adotado $(\mathrm{p}<0,05)$. Todas as análises estatísticas foram realizadas com o programa computacional Stata v.10 (StataCorp LP, College Station) para Macintosh. 


\section{Resultados}

Foram excluídos 77 pacientes que tiveram descrição incompleta ou conflitante dos quesitos pré-estabelecidos. Foram excluídos 13 pacientes que receberam tratamento hormonal neoadjuvante e 2 pacientes cujo diagnóstico foi realizado através de cirurgia endoscópica da próstata, totalizando 92 casos excluídos e 1.002 incluídos no estudo.

\subsection{Validação dos nomogramas de Crippa e Srougi}

A tabela 1 apresenta os dados demográficos com as características clínicas e patológicas dos pacientes deste estudo, confrontados com os dados dos pacientes do estudo de Crippa e Srougi. Diferenças estatisticamente significativas foram observadas em relação à idade, estadio clínico, estadio patológico, escore de Gleason (categorias 7 e 8-10), número total de fragmentos retirados e PFPB.

Os Anexos C.1 e C.2 apresentam os dois nomogramas de Crippa e Srougi para predição de DOC, que diferem entre si na forma de categorizar o escore de Gleason.

As figuras 1 e 2 apresentam as curvas ROC das predições obtidas dos dois nomogramas de Crippa e Srougi em relação aos desfechos observados nos pacientes da população de validação. ASC e os respectivos intervalos de confiança de $95 \%$ para as predições baseadas nos nomogramas 1 e 2 foram 0,68 (IC: 0,65 - 0,70) e 0,68 (IC: 0,65 - 0,71), respectivamente. O escore de Brier foi igual a 0,17 para ambos os nomogramas. 
Tabela 1: Características demográficas dos pacientes do presente estudo e do estudo de Crippa e Srougi

\begin{tabular}{|c|c|c|c|c|c|}
\hline \multirow{4}{*}{$\begin{array}{l}\text { Características } \\
\mathbf{N}^{0} \text { de pacientes } \\
\text { Idade } \\
\text { Média (mediana) }\end{array}$} & \multicolumn{2}{|c|}{ USP ${ }^{1}$} & \multicolumn{2}{|c|}{ Crippa e Srougi $^{2}$} & \multirow[t]{2}{*}{$\mathbf{p}$} \\
\hline & \multicolumn{2}{|c|}{1002} & \multicolumn{2}{|c|}{898} & \\
\hline & & & & & \\
\hline & 64,7 & $(65)$ & 62,9 & $(63,5)$ & 0,00 \\
\hline Min-max & $44-81$ & & $40-83$ & & \\
\hline Est clínico & $\mathbf{N}^{\mathbf{O}}$ & $\%$ & $\mathbf{N}^{\mathbf{o}}$ & $\%$ & \\
\hline T1c & 627 & $62,5 \%$ & 432 & $48,1 \%$ & 0,00 \\
\hline $\mathrm{T} 2$ & 371 & $37,0 \%$ & 459 & $51,1 \%$ & 0,00 \\
\hline$-\mathrm{T} 2 \mathrm{a}$ & 248 & $24,7 \%$ & $\ldots$ & $\ldots$ & \\
\hline$-\mathrm{T} 2 \mathrm{~b}$ & 97 & $9,7 \%$ & $\ldots$ & $\ldots$ & \\
\hline$-\mathrm{T} 2 \mathrm{c}$ & 26 & $2,6 \%$ & $\ldots$ & $\ldots$ & \\
\hline $\mathrm{T} 3$ & 4 & $0,4 \%$ & 7 & $0,8 \%$ & 0,00 \\
\hline Est patológico & $\mathbf{N}^{\mathbf{0}}$ & $\%$ & $\mathbf{N}^{0}$ & $\%$ & \\
\hline T0 & 16 & $1,6 \%$ & $\ldots$ & $\ldots$ & \\
\hline $\mathrm{T} 2$ & 722 & $72,0 \%$ & 599 & $66,7 \%$ & 0,00 \\
\hline$-\mathrm{T} 2 \mathrm{a}$ & 156 & $15,5 \%$ & $\ldots$ & $\ldots$ & \\
\hline$-\mathrm{T} 2 \mathrm{~b}$ & 40 & $4,0 \%$ & $\ldots$ & $\ldots$ & \\
\hline$-\mathrm{T} 2 \mathrm{c}$ & 526 & $52,5 \%$ & $\ldots$ & $\ldots$ & \\
\hline $\mathrm{T} 3$ & 259 & $25,8 \%$ & 296 & $33,0 \%$ & 0,00 \\
\hline$-\mathrm{T} 3 \mathrm{a}$ & 157 & $15,6 \%$ & $\ldots$ & $\ldots$ & \\
\hline$-\mathrm{T} 3 \mathrm{~b}$ & 102 & $10,2 \%$ & $\ldots$ & $\ldots$ & \\
\hline $\mathrm{T} 4$ & 5 & $0,5 \%$ & 3 & $0,3 \%$ & 0,02 \\
\hline PSA & $\mathbf{N}^{\mathbf{o}}$ & $\%$ & $\mathbf{N}^{\mathbf{o}}$ & $\%$ & \\
\hline Média (mediana) & 10,36 & $(9,90)$ & 10,1 & $(8,0)$ & 0,44 \\
\hline Min-max & $0,1-61,2$ & & $0,3-63,5$ & & \\
\hline $0-4$ & 111 & $11,1 \%$ & 84 & $9,4 \%$ & 0,23 \\
\hline $4,1-10$ & 510 & $50,9 \%$ & 512 & $57,0 \%$ & 0,07 \\
\hline $10,1-20$ & 295 & $29,4 \%$ & 236 & $26,3 \%$ & 0,13 \\
\hline$>20$ & 86 & $8,6 \%$ & 66 & $7,3 \%$ & 0,28 \\
\hline Gleason & $\mathbf{N}^{\mathbf{o}}$ & $\%$ & $\mathbf{N}^{\mathbf{0}}$ & $\%$ & \\
\hline $2-6$ & 711 & $71,0 \%$ & 635 & $72,7 \%$ & 0,41 \\
\hline 7 & 226 & $22,6 \%$ & 165 & $18,4 \%$ & 0,02 \\
\hline $8-10$ & 65 & $6,5 \%$ & 80 & $8,9 \%$ & 0,04 \\
\hline$N^{0}$ total frag & $\mathbf{N}^{\mathbf{0}}$ & & $\mathbf{N}^{\mathbf{o}}$ & & \\
\hline Média (mediana) & 11,6 & $(12,0)$ & 8,1 & $(7,0)$ & 0,00 \\
\hline Min-max & $2-30$ & $\ldots$ & $2-22$ & $\ldots$ & \\
\hline$N^{0}$ frag + & $\mathbf{N}^{\mathbf{0}}$ & & $\mathbf{N}^{0}$ & & \\
\hline Média (mediana) & 3,9 & $(3,0)$ & 3,2 & $(3,0)$ & 0,00 \\
\hline Min-max & $1-22$ & $\ldots$ & $1-20$ & $\ldots$ & \\
\hline PFPB & $\mathbf{N}^{\mathbf{O}}$ & & $\mathbf{N}^{\mathbf{O}}$ & & \\
\hline Média (mediana) & $34,50 \%$ & $(33,3 \%)$ & $41,20 \%$ & $(33,3 \%)$ & 0,02 \\
\hline Min-max & $3-100$ & $\ldots$ & $5-100$ & $\ldots$ & \\
\hline
\end{tabular}




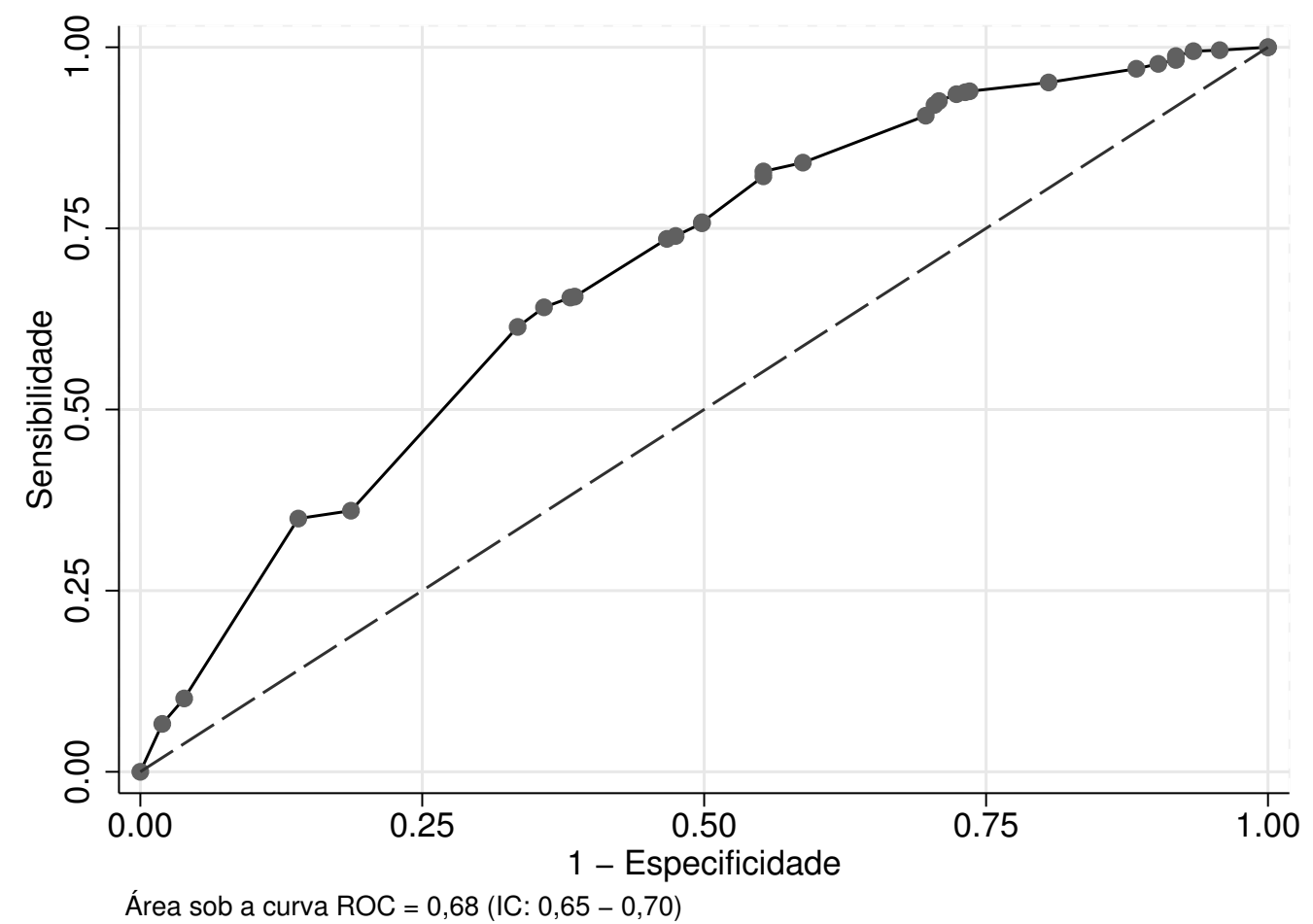

Figura 1: Curva de características operacionais - Nomograma 1 de Crippa e Srougi

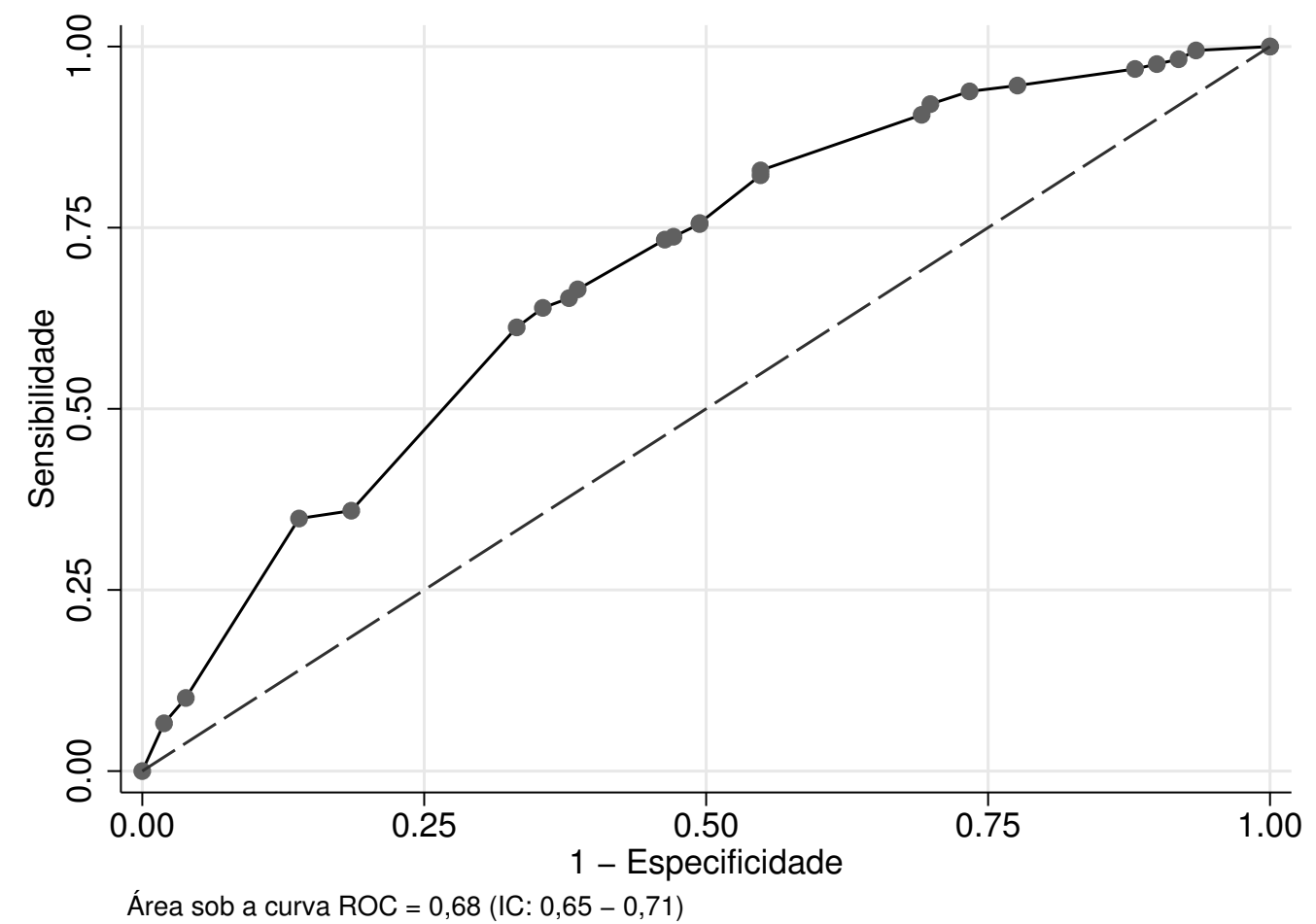

Figura 2: Curva de características operacionais - Nomograma 2 de Crippa e Srougi 
As figuras 3 e 4 demonstram graficamente a calibração dos nomogramas de Crippa e Srougi por curvas LOESS quando aplicados aos casos do presente estudo. A linha sólida da representação gráfica demonstra a considerável subestimação de DOC, em todas as faixas de predição, comparada com a linha pontilhada que demarca predição ideal.

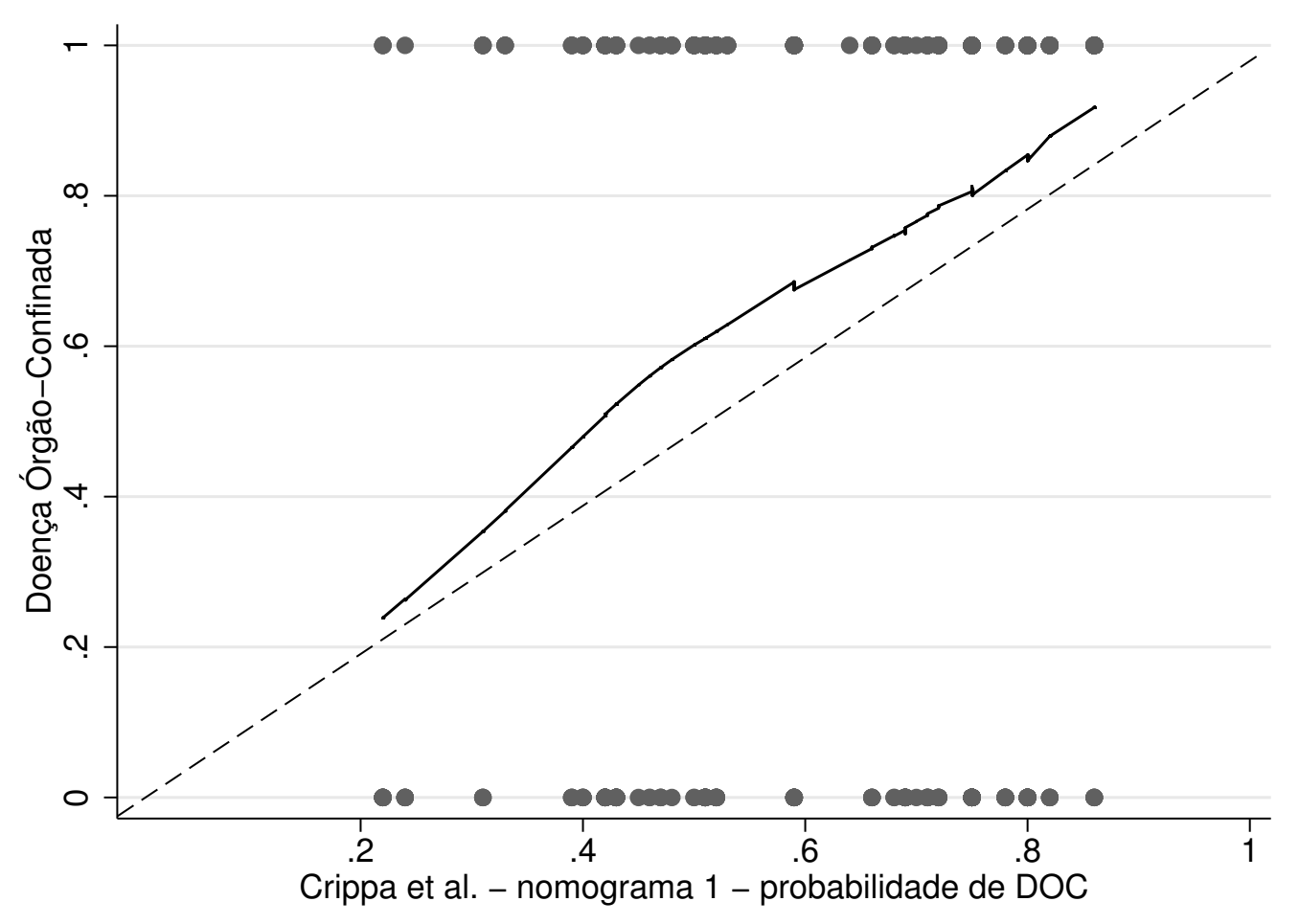

Figura 3: Distribuição das probabilidades por curvas LOESS para DOC - gráfico de calibração - Nomograma 1 de Crippa e Srougi 


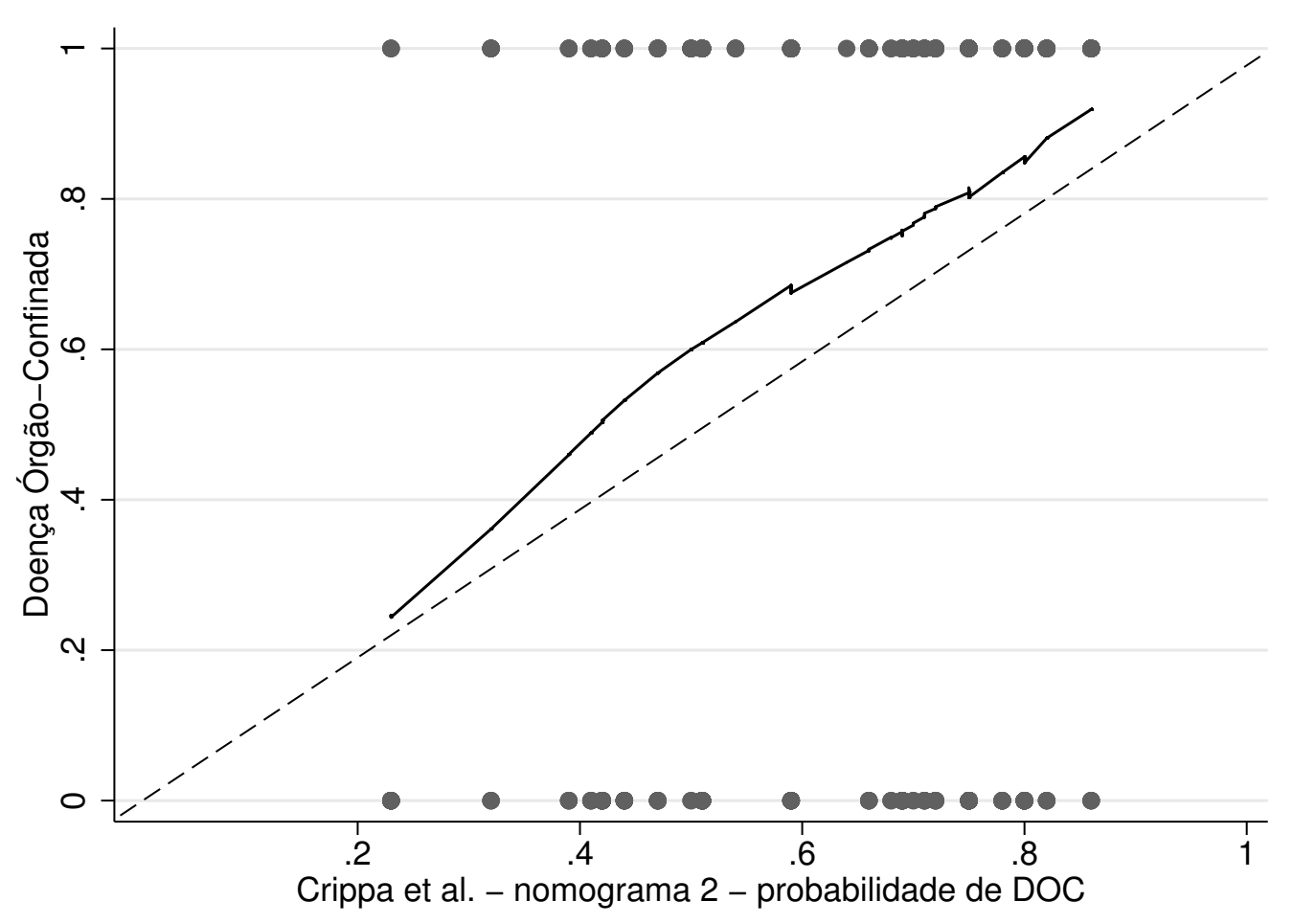

Figura 4: Distribuição das probabilidades por curvas LOESS para DOC - gráfico de calibração - Nomograma 2 de Crippa e Srougi

\subsection{Construção dos nomogramas da USP}

A Tabela 2 apresenta os resultados dos testes de qui-quadrado de Pearson usados na análise univariada. Não houve associação significativa com DOC ou IVS para o número total de fragmentos nas biópsias prostáticas e para a idade dos pacientes. As categorias das variáveis PSA, escore de Gleason, PFPB e estadio clínico foram significativamente associadas com ambos os desfechos.

A Tabela 3 representa a análise multivariada para as variáveis que se mostraram significativamente associadas aos desfechos DOC e IVS. O PSA apresentou significância estatística na sua relação com os desfechos em todas as categorias, com 
exceção da categoria 4,1-10 quando comparada com a categoria 0-4 na análise multivariada, evidenciando ausência de diferença entre esses dois grupos em sua relação com DOC e IVS. A fusão dessas duas categorias conferiu representatividade estatística no modelo final, conforme demonstra a Tabela 4. O escore de Gleason na análise multivariada apresentou correlação com os desfechos em todas as categorias, com exceção da categoria de Gleason 7, quando comparada com 8-10. A fusão dessas duas categorias conferiu representatividade estatística, conforme demonstra a Tabela 4. A PFPB na análise multivariada mostrou correlação em todos as categorias, com exceção da categoria 50,1\%-75\%, quando comparada com a categoria 0\%-25\%. A fusão das categorias $25,1 \%-50 \%$ com a categoria 50,1\%-75\% conferiu representatividade estatística, conforme demonstra a Tabela 4.

Tabela 2: Análise univariada para predição de DOC e IVS

\begin{tabular}{lccccc}
\hline & \multicolumn{2}{c}{ DOC } & \multicolumn{3}{c}{ IVS } \\
\hline Variável & $\chi^{2}$ & $\mathbf{p}$ & $\mathbf{g l}^{*}$ & $\chi^{2}$ & $\mathbf{p}$ \\
& & & & & \\
Idade & 28,3 & 0,81 & 36 & 21,6 & 0,97 \\
PSA & 67,8 & 0,00 & 3 & 65,2 & 0,00 \\
Estadio Clínico & 34,68 & 0,00 & 4 & 29,1 & 0,00 \\
No total de fragmentos & 20,9 & 0,58 & 23 & 17,49 & 0,78 \\
PFPB & 59,1 & 0,00 & 3 & 57,1 & 0,00 \\
escore de Gleason & 48,4 & 0,00 & 2 & 39,9 & 0,00 \\
\hline
\end{tabular}

* graus de liberdade

A variável estadio clínico foi rejeitada na análise multivariada com $\mathrm{p}=0,08$ para predição de DOC e 0,15 para predição de IVS. 
A Tabela 4 sumariza os modelos finais de regressão logística para predição de DOC e IVS, apresentando as razões de chance com seus respectivos intervalos de confiança de 95\%, calculados em 1.000 amostras extraídas da coorte original pelo método de bootstrap. São apresentados também os coeficientes de regressão bem como os resultados do teste de Hosmer \& Lemeshow para cada um dos modelos.

Tabela 3: Análise multivariada para predição de DOC e IVS

\begin{tabular}{|c|c|c|c|c|c|c|}
\hline & & DOC $^{1}$ & & & IVS $^{2}$ & \\
\hline & $\mathbf{O R}^{3}$ & $95 \%$ IC & $\mathbf{p}$ & $\mathbf{O R}^{3}$ & $95 \%$ IC & $\mathbf{p}$ \\
\hline PSA & & & $\mathbf{0 , 0 0}$ & & & $\mathbf{0 , 0 0}$ \\
\hline $4,1-10$ versus $0-4$ & 0,73 & {$[0,39-1,36]$} & 0,24 & 0,88 & {$[0,34-2,28]$} & 0,79 \\
\hline $10,1-20$ versus $0-4$ & 0,36 & {$[0,19-0,68]$} & 0,00 & 2,90 & {$[1,16-7,28]$} & 0,02 \\
\hline$>20$ versus $0-4$ & 0,18 & {$[0,09-0,38]$} & 0,0 & 4,79 & {$[1,76-13,04]$} & 0,00 \\
\hline Gleason & & & $\mathbf{0 , 0 0}$ & & & $\mathbf{0 , 0 0}$ \\
\hline 7 versus 4-6 & 0,51 & {$[0,36-0,73]$} & 0,00 & 2,10 & {$[1,30-3,41]$} & 0,00 \\
\hline 7 versus $8-10$ & 1,33 & {$[0,73-2,42]$} & 0,34 & 0,71 & {$[0,35-1,44]$} & 0,35 \\
\hline $8-10$ versus $4-6$ & 0,38 & {$[0,22-0,68]$} & 0,00 & 2,95 & {$[1,49-5,83]$} & 0,00 \\
\hline$\%$ fragmentos positivos & & & $\mathbf{0 , 0 0}$ & & & $\mathbf{0 , 0 0}$ \\
\hline $25,1-50$ versus $0-25$ & 0,56 & {$[0,39-0,80]$} & 0,00 & 1,57 & {$[0,91-2,73]$} & 0,10 \\
\hline $50,1-75$ versus $0-25$ & 0,68 & {$[0,39-1,19]$} & 0,18 & 2,22 & {$[1,06-4,63]$} & 0,03 \\
\hline $75,1-100$ versus $0-25$ & 0,30 & {$[0,17-0,53]$} & 0,00 & 4,15 & {$[2,06-8,39]$} & 0,00 \\
\hline Estadio clínico & & & $\mathbf{0 , 0 8}$ & & & 0,15 \\
\hline $\mathrm{T} 2 \mathrm{a}$ versus $\mathrm{T} 1 \mathrm{c}$ & 0,68 & {$[0,47-0,97]$} & 0,03 & 1,86 & {$[1,13-3,05]$} & 0,01 \\
\hline $\mathrm{T} 2 \mathrm{~b}$ versus $\mathrm{T} 1 \mathrm{c}$ & 0,59 & {$[0,35-0,98]$} & 0,04 & 1,50 & {$[0,74-3,01]$} & 0,25 \\
\hline $\mathrm{T} 2 \mathrm{c}$ versus $\mathrm{T} 1 \mathrm{c}$ & 0,46 & {$[0,18-1,11]$} & 0,08 & 2,02 & {$[0,69-5,93]$} & 0,19 \\
\hline $\mathrm{T} 3$ versus $\mathrm{T} 1 \mathrm{c}$ & 0,75 & {$[0,98-5,89]$} & 0,79 & 1,09 & {$[1,00-12,13$} & 0,93 \\
\hline
\end{tabular}


Tabela 4: Modelos finais de regressão logística para predição de DOC e IVS

\begin{tabular}{lcccc}
\hline & \multicolumn{4}{c}{ Doença Órgão-Confinada } \\
& coef $^{1}$ & OR $^{2}$ & IC 95\% & p \\
\hline PSA & & & & $\mathbf{0 , 0 0}$ \\
$10,1-20$ versus 0-4 & $-0,74$ & 0,48 & {$[0,35-0,66]$} & 0,00 \\
$>20$ versus 0-4 & $-1,43$ & 0,24 & {$[0,15-0,39]$} & 0,00 \\
Gleason 7-10 versus 4-6 & $-0,75$ & 0,47 & {$[0,34-0,65]$} & 0,00 \\
PFPB & & & & $\mathbf{0 , 0 0}$ \\
$25,1-75$ versus 0-25 & $-0,62$ & 0,54 & {$[0,39-0,74]$} & 0,00 \\
$75,1-100$ versus 0-25 & $-1,39$ & 0,25 & {$[0,14-0,43]$} & 0,00 \\
Constante & 2,14 & 8,47 & - & 0,00 \\
\hline Valor preditivo positivo: $77,03 \%$ & \multicolumn{4}{c}{ Valor preditivo negativo: $59,49 \%$} \\
Hosmer and Lemeshow: $\chi^{2}=7,9$ gl=6 $\mathrm{p}=0,24$ \\
\hline
\end{tabular}

\begin{tabular}{|c|c|c|c|c|}
\hline & \multicolumn{4}{|c|}{ Invasão de Vesículas Seminais } \\
\hline & $\operatorname{coef}^{1}$ & $\mathbf{O R}^{2}$ & IC 95\% & $\mathbf{p}$ \\
\hline PSA & & & & $\mathbf{0 , 0 0}$ \\
\hline $10,1-20$ versus $0-4$ & 1,17 & 3,23 & {$[2,00-5,24]$} & 0,00 \\
\hline$>20$ versus $0-4$ & 1,74 & 5,67 & {$[3,08-10,44]$} & 0,00 \\
\hline Gleason 7-10 versus 4-6 & 0,86 & 2,37 & {$[1,53-3,67]$} & 0,00 \\
\hline PFPB & & & & $\mathbf{0 , 0 0}$ \\
\hline $25,1-75$ versus $0-25$ & 0,65 & 1,92 & {$[1,16-3,20]$} & 0,01 \\
\hline $75,1-100$ versus $0-25$ & 1,64 & 5,15 & {$[2,63-10,09]$} & 0,00 \\
\hline Constante & $-3,70$ & 0,025 & - & 0,03 \\
\hline \multicolumn{2}{|l|}{ Valor preditivo positivo: $42,86 \%$} & \multicolumn{3}{|c|}{ Valor preditivo negativo: $89,53 \%$} \\
\hline \multicolumn{5}{|c|}{ Hosmer and Lemeshow: $\chi^{2}=3,9 \mathrm{gl}=6 \mathrm{p}=0,67$} \\
\hline
\end{tabular}


A tabela 5 apresenta o nomograma da USP para predição de DOC.

Tabela 5: Nomograma USP: Predição de DOC

\begin{tabular}{|c|c|c|c|}
\hline & \multicolumn{3}{|c|}{$\begin{array}{c}\text { Gleason 4-6 } \\
\text { PSA }\end{array}$} \\
\hline PFPB & 0 - 10 & $10,1-20$ & $>\mathbf{2 0}$ \\
\hline $0-25 \%$ & $89[84,5-92,1]$ & $80[70,2-86,5]$ & $67[46,0-84,6]$ \\
\hline $25,1-75 \%$ & $81[74,8-85,8]$ & $68[57,5-76,5]$ & $52[32,3-71,6]$ \\
\hline \multirow[t]{2}{*}{$75,1-100 \%$} & $68[42,3-87,3]$ & $50[23,2-70,8]$ & $33[8,3-64,2]$ \\
\hline & & $\begin{array}{c}\text { Gleason 7-10 } \\
\text { PSA }\end{array}$ & \\
\hline PFPB & 0 - 10 & $10,1-20$ & $>20$ \\
\hline $0-25 \%$ & $80[67,2-87,7]$ & $65[46,9-78,8]$ & $49[11,7-76,9]$ \\
\hline $25,1-75 \%$ & $68[57,3-77,7]$ & $50[37,1-62,8]$ & $34[18,6-52,1]$ \\
\hline $75,1-100 \%$ & $50[27,3-68,2]$ & $32[11,7-54,6]$ & $19[2,5-51,3]$ \\
\hline
\end{tabular}

A tabela 6 apresenta o nomograma da USP para predição de IVS.

Tabela 6: Nomograma USP: Predição de IVS

\begin{tabular}{|c|c|c|c|}
\hline & \multicolumn{3}{|c|}{$\begin{array}{c}\text { Gleason 4-6 } \\
\text { PSA }\end{array}$} \\
\hline PFPB & 0 - 10 & $10,1-20$ & $>\mathbf{2 0}$ \\
\hline $0-25 \%$ & $2,4[1,0-4,9]$ & $7,4[3,6-14,7]$ & $12,3[2,9-31,3]$ \\
\hline $25,1-75 \%$ & $4,5[2,4-8,5]$ & $13,3[7,8-22,1]$ & $21,3[7,6-40,4]$ \\
\hline \multirow[t]{2}{*}{$75,1-100 \%$} & $11,3[1,3-33,3]$ & $29,2[12,6-57,6]$ & $42,0[15,8-74,9]$ \\
\hline & & $\begin{array}{c}\text { Gleason 7-10 } \\
\text { PSA }\end{array}$ & \\
\hline PFPB & 0 - 10 & $10,1-20$ & $>\mathbf{2 0}$ \\
\hline $0-25 \%$ & $5,5[1,7-14,1]$ & $16,0[7,1-32,6]$ & $25,0[3,6-62,4]$ \\
\hline $25,1-75 \%$ & $10,1[5,4-19,4]$ & $26,8[16,1-38,9]$ & $39,1[21,5-55,7]$ \\
\hline $75,1-100 \%$ & $23,2[8,5-43,3]$ & $49,5[26,7-73,2]$ & $63,2[25,0-84,1]$ \\
\hline
\end{tabular}


A validação interna foi realizada por meio de bootstrap com 1.000 amostras e os resultados de desempenho e acurácia foram aferidos com a mesma metodologia aplicada na validação dos nomogramas de Crippa e Srougi.

As figuras 5 e 6 apresentam as curvas ROC que ilustram a capacidade de discriminação dos nomogramas da USP nas amostras de bootstrap para DOC e IVS, respectivamente. As áreas sob a curva ROC para os nomogramas de predição de DOC e de IVS foram de 0,73 (IC: 0,70 - 0,76) e 0,77 (IC: 0,73 - 0,79), respectivamente. Os nomogramas da USP apresentaram escores de Brier de 0,16 e 0,08, respectivamente.

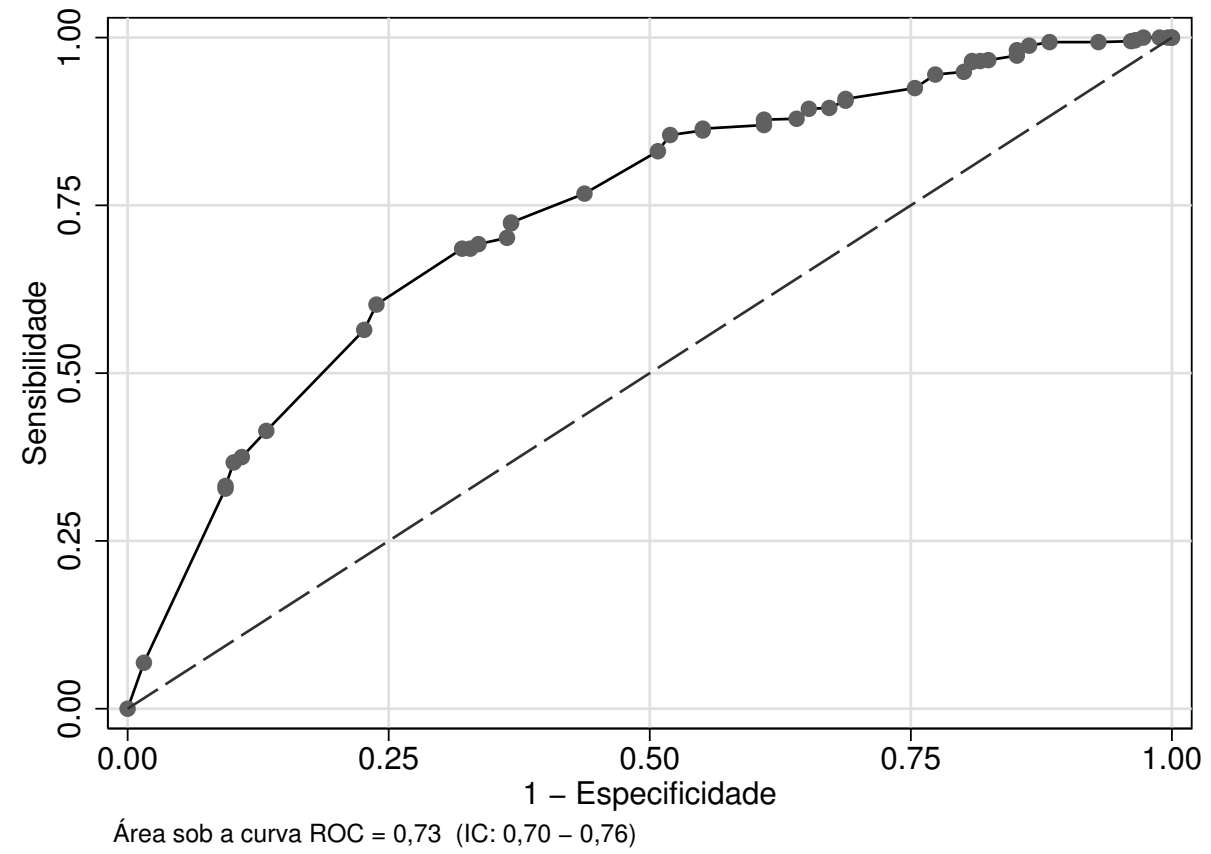

Figura 5: Curva de características operacionais do nomograma da USP para predição de DOC em 1.000 amostras de bootstrap 


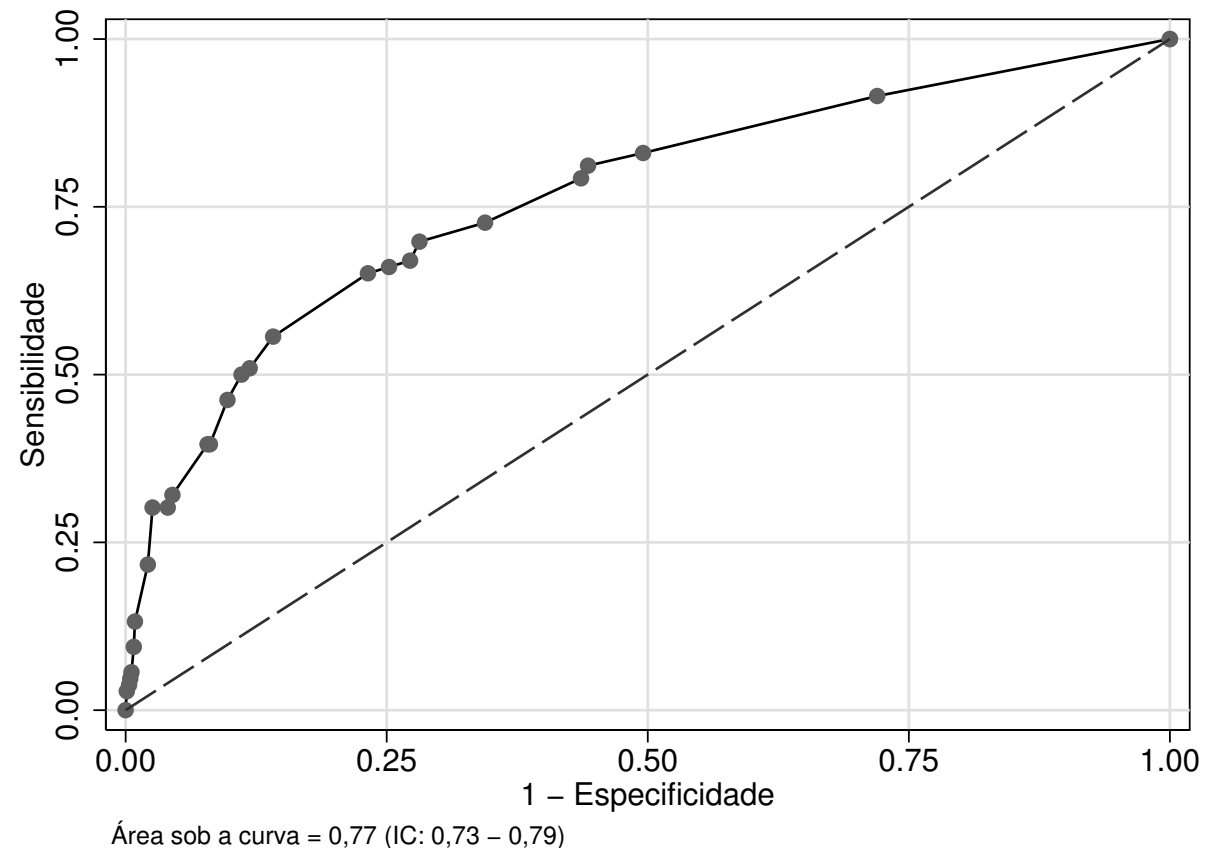

Figura 6: Curva de características operacionais do nomograma da USP para predição de IVS em 1.000 amostras de bootstrap

As curvas LOESS de distribuição ilustradas nas figuras 7 e 8 representam graficamente a calibração dos nomogramas da USP em relação às faixas de predição. $O$ nomograma para predição de DOC apresentou curva coincidente com a linha média, denotando boa calibração em todas os segmentos, com exceção da faixa entre $15 \%$ e $30 \%$, onde o nomograma da USP superestima a DOC. O nomograma para predição de IVS superestima o desfecho em todas as faixas de predição acima de $2 \%$. 


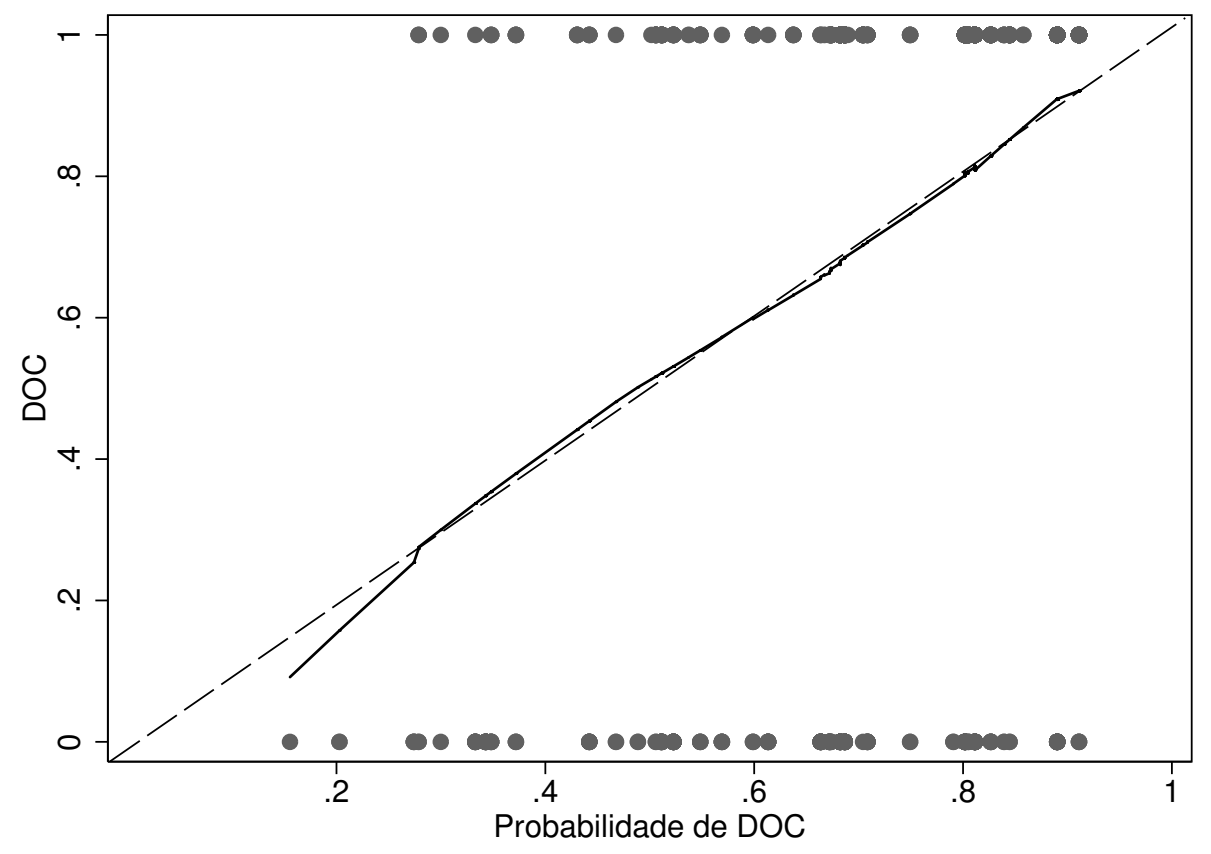

Figura 7: Distribuição das probabilidades por curvas LOESS para DOC - gráfico de calibração - Nomograma da USP em 1.000 amostras de bootstrap

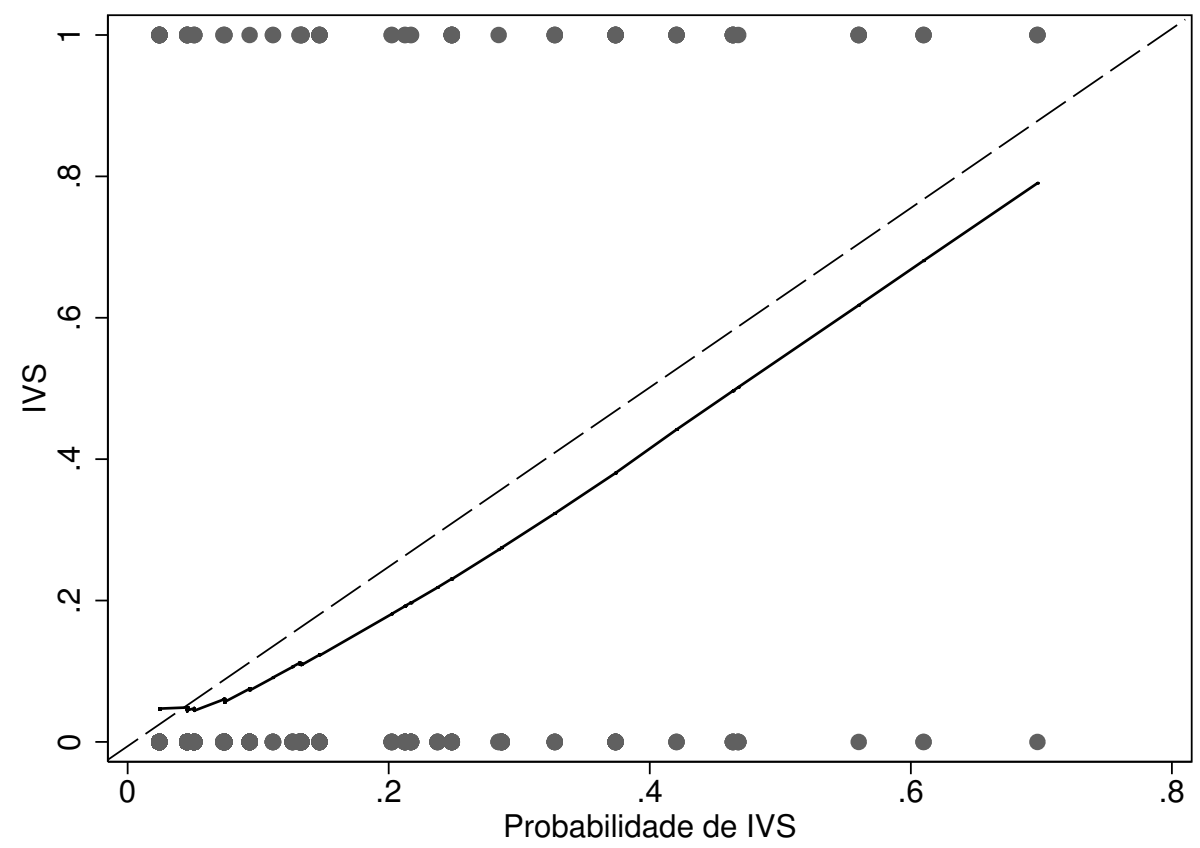

Figura 8: Distribuição das probabilidades por curvas LOESS para IVS - gráfico de calibração - Nomograma da USP em 1.000 amostras de bootstrap 


\section{Discussão}

O valor de um modelo matemático de predição na área médica não se baseia na sua complexidade, elegância ou proliferação, mas sim na sua utilidade clínica.

Este estudo validou externamente em ambiente docente de uma instituição assistencial pública um nomograma com grande apelo à simplicidade de uso, construído originalmente em população brasileira, em ambiente controlado de uma instituição assistencial de caráter privado. Na sequência, o presente estudo desenvolveu os nomogramas da USP com base no ajustamento e reagrupamento das mesmas variáveis, resultando em dois nomogramas ainda mais concisos de predição para dois desfechos: DOC e IVS.

A desafiadora tarefa de informar e aculturar um paciente a respeito de sua doença por vezes alcança grande complexidade, independente do contexto cultural do mesmo. Diante do homem simples, por vezes analfabeto, depara-se o médico com a indispensável necessidade de simplificar conceitos, lançar mão de exemplos e comparações do cotidiano, e trazer seu discurso o mais próximo possível do patamar de seu interlocutor. Diante do homem letrado, muitas vezes conhecedor de nuances de anatomia e de fisiopatologia da neoplasia, quando não colega médico, encontra-se o urologista com a missão de embasar na literatura e na evidência científica os conceitos e condutas apresentados. O cenário existente entre esses dois extremos é composto por um leque de possibilidades de compreensão e de argumentação. 
A construção da decisão busca colocar o paciente em condições de manifestar-se sobre as alternativas de tratamento e obedece um processo que, se bem instrumentalizado, pode facilitar sobremaneira sua compreensão dos fatos. O paciente com câncer de próstata clinicamente localizado tem diante de si um dilema decisório em que, de um lado, pesa o medo da morte e do sofrimento com a doença avançada, que poderão advir se o tratamento for inadequado e, de outro, o difundido medo da impotência sexual. Essa decisão só se torna menos complexa na medida em que o paciente constrói, em seu pensamento, figuras comparáveis relacionadas às vantagens e desvantagens de cada tratamento proposto. Um nomograma de fácil compreensão por parte do indivíduo leigo que lhe diga quais as suas chances de ter doença realmente restrita à próstata torna-se um instrumento de grande valor na mão de um médico atencioso que ajuda seu paciente nesse angustiante caminho.

Dentro das instituições de referência, onde o grande número de atendimentos diários e a grande complexidade dos casos domina o cenário ambulatorial a simplicidade de um nomograma é fator condicionante de seu uso na prática.

A natureza retrospectiva deste estudo pode ter acarretado a existência de alguns vieses no que diz respeito às variáveis preditoras. O estadiamento dos pacientes obedecendo níveis de evidência, principalmente em instituições de ensino e de assistência pública, não permite a realização de exames de imagem para pacientes de baixo risco e, portanto, o toque retal é a única informação registrável para definir o estadio clínico. No HCFMUSP/ICESP o toque retal é realizado pelo médico residente de urologia, sob supervisão. Isso traz uma inerente variabilidade de interpretação que é observador dependente e não tem como ser ponderada retrospectivamente. De um ponto de vista isso constitui um importante viés para essa variável, mas de outro ponto de vista essa é a situação real de atendimento nos hospitais de ensino e de referência, campo final de atuação para qualquer ferramenta de predição. Nessa mesma linha de ponderação, o PSA e os dados da biópsia prostática merecem considerações. 
O PSA trazido pelo paciente, juntamente com o laudo da biópsia prostática de sua instituição de origem foram considerados neste estudo. No momento da programação cirúrgica, o PSA foi refeito no HCFMUSP para os pacientes em que o exame original tinha mais de 3 meses. A biópsia não foi refeita em nenhum paciente. É ponderável que a falta de consistência de método para a realização dessas duas variáveis de grande peso constitui uma fragilidade no presente estudo, no entanto, essa dinâmica espelha exatamente a realidade das instituições de referência, que recebem o paciente já biopsiado. A biópsia de próstata não é isenta de riscos. Numa revisão de 5802 biópsias de próstata, hematuria e/ou hematospermia ocorreram em aproximadamente 50\% dos pacientes e persistiram por mais de 3 dias em $23 \%$ deles $^{44}$. A infecção urinária ocorre em até $11 \%$ dos pacientes biopsiados, podendo evoluir para sepse em até $3 \%$ destes $^{45}$. Portanto, não se encontra justificativa ética para repetir a biópsia que não a clara dúvida diagnóstica. Essa dúvida não existe quando o laudo apresentado pelo paciente descreve categoricamente a presença da neoplasia e estabelece a classificação de Gleason de forma adequada.

O tratamento cirúrgico dos pacientes deste estudo, realizado por médicos residentes da disciplina de Urologia do HCFMUSP, obedeceu em todos os casos a metodologia instituída no HCFMUSP e ICESP pelo Professor Titular da disciplina, rigorosamente seguida por todos os urologistas supervisores dos residentes em treinamento nestas instituições. Nesse aspecto, apesar da inerente diferença de nível técnico entre os cirurgiões em treinamento, própria do ambiente docente, a supervisão e a técnica cirúrgica empregada são rigidamente norteadas e conferem a necessária consistência de metodologia.

Também o estudo da peça cirúrgica na casuística deste estudo apresentou variabilidade no que se refere ao patologista responsável pelo estabelecimento dos critérios de desfecho. Os exames foram realizados e registrados, sob supervisão, pelos médicos residentes de anatomia patológica e o laudo oficialmente registrado no prontuário do 
paciente foi utilizado para definir os critérios de desfecho. Novamente, essa inconsistência pode ser interpretada como fragilidade no estudo, porém, representa de forma inequívoca a dinâmica de trabalho das instituições terciárias de ensino.

Estas são, na verdade, as diferenças mais importantes entre as populações do estudo de Crippa e Srougi e deste estudo. Em que pese que os locais de atendimento das populações são geograficamente similares, na essência da coleta das variáveis, no aspecto sócio-assistencial, e na condução cirúrgica elas são muito distintas, o que confere grande peso à validação externa aqui apresentada. A multiplicidade de origem nas variáveis aqui estudadas é parte inerente de outros estudos de validação de grande abrangência, como o estudo de coorte do próprio Partin, validando seu nomograma na base de dados da The National Cancer Institute Surveillance, Epidemiology and End Results (SEER), amostra nacional nos Estados Unidos que reúne pacientes de instituições dos mais variados níveis assistenciais. Vale ressaltar que naquele importante trabalho de validação das tabelas de predição de Partin, o uso de terapia hormonal neoadjuvante não pode ser ponderado e usado como critério de exclusão porque a base de dados não especifica essa informação ${ }^{46}$.

As curvas ROC das figuras 1 e 2 e respectivas ASC das probabilidades de Crippa e Srougi aplicadas aos pacientes deste estudo sugerem capacidade de estratificação moderada, com ASC de 0,68 para ambos os nomogramas. Isso equivale a dizer que aqueles nomogramas classificaram equivocadamente $32 \%$ dos pacientes deste estudo com relação à condição de DOC. Como forma de estimar o desempenho geral dos nomogramas foram calculados os escores de Brier, que representam o desvio quadrado médio entre as probabilidades preditas e as taxas de DOC reais observadas. Os dois escores foram de 0,17 , sendo que a interpretação desse escore define 0 como indicando perfeito desempenho informativo do modelo e 0,25 como ausência total de informação, ou em outros termos, o equivalente ao acaso definido pelo cara ou coroa de uma moeda. 
Apesar de bem representar a capacidade discriminativa, nenhum desses testes demonstra a isoladamente calibração dos nomogramas em validação de acordo com cada patamar de risco predito. Para isso as curvas LOESS ou curvas de calibração exploram graficamente essa característica e as figuras 3 e 4 mostram claramente a condição de subestimação que ocorreu em todas as faixas de predição, em ambos os nomogramas.

A persistência em uso das tabelas de Partin espelha a importância clínica de um modelo com grande valor prognóstico intrínseco, útil mesmo em contextos onde sua validade estatística foi modestamente validada ${ }^{47}$. Por esse prisma, mesmo com os resultados de acurácia e desempenho modestos para os nomogramas de Crippa e Srougi no presente estudo, a importância intrínseca de sua informação prognóstica por trazer uma variável alternativa ao estadio clínico lhe confere grande potencial de validade clínica. A performance preditiva moderada de tais nomogramas pode ter sido influenciada por vieses relacionados às variáveis preditoras, reforçando a hipótese original deste estudo de que a heterogeneidade das variáveis, inerente aos centros terciários de ensino, poderia revelar fragilidades de uma ferramenta de predição desenvolvida em uma população mais homogênea e em ambiente controlado no que se refere à origem das variáveis preditoras. O próximo passo lógico no estudo foi criar novos nomogramas derivados das mesmas variáveis, porém, desenvolvidos e validados na população dessa instituição terciária de ensino, incluindo um modelo preditor para IVS.

O processo de validação externa, especialmente de domínio, de uma ferramenta de predição quando conduzido de forma metodologicamente apropriada e ajustado para acomodar as inerentes diferenças populacionais determina, no conjunto de seus resultados, uma fenômeno de grande relevância: a mudança de nível de evidência. Segundo McGinn et al. ${ }^{32}$ esse processo traz a ferramenta de predição em estudo do nível 4 para o nível 2 de evidência, com claras implicações no peso decisório e no respaldo oferecido por tal ferramenta. Na verdade, a autorização para uso clínico depende diretamente desse quesito. A validação externa é o único procedimento dentro do complexo 
sistema de construção de um nomograma que está diretamente focado na sua performance fora do seu contexto original. Sabemos da condição utópica de sonharmos com uma ferramenta de predição ideal, com área sob a curva igual a 1 e escore de Brier igual a zero. No entanto, a performance conseguida, quando estatisticamente significativa e reprodutível em condições essencialmente distintas (validada externamente), é o que de melhor o universo da atividade médica consegue produzir, dado suas óbvias limitações impostas pela ética e pelo respeito ao ser humano.

Os nomogramas da USP exibem consistência, na medida em que os intervalos de confiança das razões de chance observados na amostra que deu origem aos mesmos coincidem com os intervalos de confiança obtidos pela técnica de bootstrap e exibem adequada performance preditiva demonstrada pelo teste de Hosmer \& Lemeshow.

O nomograma da USP para predição de DOC aplicado a 1.000 amostras de bootstrap apresentou discriminação melhor do que os nomogramas de Crippa e Srougi, conforme demonstra a curva ROC da figura 5 com ASC de 0,73 (IC: 0,70-0,76), mostrando correta classificação em $73 \%$ dos casos. O desempenho geral do nomograma pode ser considerado moderado com escore de Brier de 0,16. A curva LOESS ou gráfico de calibração na figura 7, no entanto, mostra calibração bastante próxima do ideal em todas as faixas de predição de DOC, com discreta tendência de superestimação na faixa entre $15 \%$ e $30 \%$, o que confere considerável confiabilidade às probabilidades estimadas pelo nomograma da USP para DOC quando aplicadas à mesma população.

O nomograma da USP para predição de IVS apresentou considerável capacidade discriminativa, conforme demonstra a ASC de 0,77 (IC: 0,73-0,79) na curva ROC da Figura 6, mostrando correta classificação de 77\% dos casos, e o escore de Brier de 0,08 indica bom desempenho informativo do nomograma. A análise de calibração desse nomograma na curva LOESS, no entanto, mostra clara superestimação do desfecho em todas as faixas de predição acima de $2 \%$. 
O comportamento superotimista das ferramentas de predição nos processos de validação é um fenômeno comum e bem conhecido ${ }^{48}$. Justice afirma que a adequada calibração de um nomograma é mais útil ao médico do que a capacidade de discriminação durante o processo de tomada de decisão ${ }^{36}$. Dessa forma, após validar externamente os nomogramas de Crippa e Srougi com as mesmas variáveis, na nossa visão o nomograma da USP para predição de DOC já encontra suporte suficiente para ser utilizado na população do HCFMUSP/ICESP, haja vista a excelente calibração e boa discriminação obtidos com o ajuste de variáveis realizado neste estudo. O nomograma da USP para predição de IVS necessita ainda extensa validação externa e ajuste das variáveis para aumentar sua acurácia.

Até hoje as tabelas de Partin são utilizadas e validadas em vários centros do mundo ${ }^{23,46,49-52}$ demonstrando claro interesse da comunidade urológica em ferramentas que estimam a probabilidade de DOC. Apesar de boa capacidade discriminativa com ASC acima de 0,7 em recente validação realizada com a base de dados SEER, nos Estados Unidos, aquele estudo não reportou a calibração dos nomogramas de $\operatorname{Partin}^{46}$. Contrariamente, um estudo de validação das tabelas de Partin em população Europeia evidenciou pobre capacidade discriminativa e calibração inadequada ${ }^{47}$. Para justificar esses resultados conflitantes, inferimos como causa o fato de que, em 1993, a variável estadio clínico que Partin estudou apresentava representatividade diferente, haja vista que àquela época apenas $39 \%$ dos pacientes tinham toque retal normal ao diagnóstico ${ }^{53}$ e, portanto, a informação derivada do toque retal era melhor classificável do que atualmente, onde mais de $50 \%$ dos pacientes apresentam-se com esse estadio. Este estudo demonstra claramente a preponderância do estadio clínico T1c no momento do diagnóstico.

A crescente incidência de tumores $\mathrm{T} 1 \mathrm{c}$ tem registro claro na literatura ${ }^{54}$. Na série de Crippa e Srougi a incidência de T1c foi de 48,1\% numa casuística de pacientes diagnosticados entre 1988 e 2002. Este estudo, com casuística mais recente, apresenta 
$62,7 \%$ de pacientes com T1c. Esses fatos justificam os ajustes repetidos e sucessivos processos de validação que são necessários para acomodar as mudanças que ocorrem ao longo do tempo, tanto nas características da doença, como nas características das populações.

Sebo et al. demonstraram, através de análise multivariada dos dados de 207 pacientes submetidos a prostatectomia radical, a clara superioridade da PFPB juntamente com o PSA e o Gleason da biópsia prostática em relação ao estadio clínico na predição de doença extra-prostática ${ }^{55}$. A hipótese inicial do estudo de Crippa e Srougi de que o estadio clínico perde importância frente à PFPB se confirma neste estudo.

Segundo Altman e Royston um nomograma com propósitos clínicos deve ser construído com o menor número de variáveis possível e resultar em uma ferramenta simples de ser utilizada, caso contrário corre o risco de não ser usada no contexto agitado da atividade assistencial $^{48}$. Segundo Steyerberg et al. a incidência do desfecho também tem importância na utilidade clínica de um nomograma ${ }^{56}$. Nesse contexto a existência de doença extra-prostática em $24,75 \%$ dos pacientes deste estudo mostra claramente a utilidade de uma ferramenta que consiga discriminar com boa precisão essa condição desfavorável, que existe em um quarto dos pacientes com doença clinicamente localizada. Ainda segundo os mesmos autores, a apresentação da probabilidade na forma de porcentagem é a que mais serve ao médico que a usa no processo decisório. Esse aspecto é especialmente importante porque as ferramentas de predição são desenvolvidas com grande apelo ao conjunto de casos, mas com limitado auxílio no nível do indivíduo, por mais acurada que sejam, principalmente quando a probabilidade de desfecho estimada para esse indivíduo está fora dos extremos e/ou a incidência do desfecho é pequena ${ }^{56}$. Os nomogramas aqui validados e ajustados cumprem exemplarmente esse papel porque utilizam apenas 3 variáveis que estão disponíveis na quase totalidade dos pacientes com diagnóstico de CaP clinicamente localizado e estão representados na forma de tabelas que expressam as porcentagens de probabilidade de desfecho. 
As técnicas de preservação dos feixes vasculonervosos dos corpos cavernosos na prostatectomia radical podem contribuir para a preservação da função erétil e melhorar a qualidade de vida após a cirurgia. No entanto, na presença de doença extra-prostática, essa abordagem pode acarretar substancial risco de margem positiva e comprometer o controle do câncer ${ }^{29}$. A técnica clássica de prostatectomia radical inclui a retirada das vesículas seminais. Alguns autores sugerem não ressecá-las como forma de minimizar o trauma cirúrgico e melhorar os resultados funcionais como função erétil e continência urinária ${ }^{57}$. Também para o radioterapeuta a confiável informação sobre o comprometimento das vesículas seminais é importante, na medida em que a área irradiada é maior quando as vesículas seminais são incluídas no campo irradiado ${ }^{58}$. No entanto, a invasão das vesículas seminais está associada a taxas mais altas de recidiva bioquímica e pior prognóstico ${ }^{59}$.

Predizer adequadamente a condição de DOC é crucial para a decisão de preservar os feixes vasculonervosos e/ou as vesículas seminais dos pacientes com indicação de tratamento cirúrgico. Em que pese que essa decisão não se restringe às informações prognósticas pré-operatórias, mas passa também pela percepção do cirurgião no transoperatório, estimar adequadamente a condição de doença órgão-confinada pode contribuir de forma importante no momento de informar ao paciente os riscos previstos com o tratamento cirúrgico. O paciente cuja probabilidade de DOC é baixa pode ser informado de sua maior probabilidade de insuficiência erétil após a cirurgia, na medida em que a chance de realização das citadas técnicas de preservação é menor.

O crescente número de pacientes com doença de baixo risco conduzidos com observação vigilante continua gerando controvérsias a respeito dos conceitos de doença indolente, critérios para tratamento e impacto na sobrevida global desses pacientes comparados com os pacientes tratados ${ }^{30}$. Nomogramas para predição de doença indolente ${ }^{60,61}$ e de sobrevida de pacientes em programas de $\mathrm{OV}^{62}$ vêm surgindo recentemente como uma alternativa na tomada de decisão de conduta para esses pacientes. 
Tais nomogramas também carecem de validação externa exaustiva para uma confiável extrapolação de seus resultados para outras populações. Este estudo apresentou 419 pacientes considerados de baixo risco que foram submetidos à cirurgia, constituindo $41,1 \%$ da população que deu origem aos nomogramas da USP. Desses, $86,4 \%$ apresentaram DOC na peça cirúrgica, sendo que o nomograma da USP para DOC previu esse desfecho em $86,1 \%$, demonstrando excelente calibração nesse grupo de pacientes. A OV prevê a realização de PSA e re-biópsias de próstata periodicamente, o que permite o recálculo da probabilidade de DOC nos nomogramas da USP para os pacientes seguidos em um programa de OV. Em que pese que essa ferramenta, por suas limitações intrínsecas já discutidas, não define por si só a conduta a ser tomada no paciente com $\mathrm{CaP}$ de baixo risco, certamente pode ser uma ferramenta útil na discussão das possibilidades com o paciente, haja vista sua simplicidade e facilidade em ser reaplicada a cada novo PSA dosado e a cada re-biópsia realizada.

O desenvolvimento de novas ferramentas de predição e a atualização das já existentes é um processo que não se extingue. O aprimoramento prospectivo dos nomogramas aqui apresentados, com a inclusão de variáveis como a presença de invasão angiolinfática e/ou perineural na biópsia prostática, bem como novos marcadores celulares, moleculares e genéticos, podem melhorar a acurácia e a transportabilidade dessas ferramentas de predição ${ }^{63}$.

Diante da boa performance do nomograma da USP para predição de DOC, obtida com o ajustamento do nomograma de Crippa e Srougi no processo de validação externa aqui desenvolvido, inferimos que o nomograma da USP para predição de DOC apresenta nível 2 de evidência segundo a classificação de McGinn et al. ${ }^{32}$ e portanto sugerimos seu uso clínico, especialmente nos ambulatórios de urologia do HCFMUSP e ICESP. 


\section{Conclusões}

- Este estudo validou externamente com sucesso os nomogramas de Crippa e Srougi, mostrando moderado poder discriminativo e considerável subestimação de DOC na sua análise de calibração.

- Este estudo desenvolveu e validou internamente os nomogramas da USP para predição de DOC e de IVS. O nomograma para predição de DOC mostrou bom poder discriminativo e excelente calibração. O nomograma para predição de IVS mostrou bom poder discriminativo e considerável superestimação de IVS na sua análise de calibração. 


\section{ANEXOA}

\section{TNM 2002}

\section{Avaliação do Tumor Primário (T)}

Tx - Tumor primário não pode ser avaliado

T0 - Sem evidência de tumor

T1 - Tumor presente mas não detectável clinicamente ou com exames de imagem

- T1a - Tumor incidentalmente encontrado em menos de 5\% de fragmentos prostáticos obtidos em tecido prostático ressecado

- T1b - Tumor incidentalmente encontrado em mais de 5\% de fragmentos prostáticos obtidos em tecido prostático ressecado

- T1c - Tumor encontrado em biópsia prostática motivada por aumento do PSA

T2 - Tumor palpável ao toque retal mas restrito aos limites da glândula prostática

- T2a - Tumor palpável em metade ou menos da metade de um dos lobos prostáticos

- T2b - Tumor palpável em mais da metade de um dos lobos prostáticos

- T2a - Tumor palpável nos dois lobos prostáticos

T3 - Tumor extra-prostático

- T3a - Tumor ultrapassa a cápsula prostática em um ou nos dois lados

- T3b - Tumor invade as vesículas seminais

\section{Avaliação dos Linfonodos}

$\mathbf{N x}$ - Linfonodos regionais não podem ser avaliados

No - Ausência de comprometimento dos linfonodos regionais

N1 - Presença de comprometimento dos linfonodos regionais 


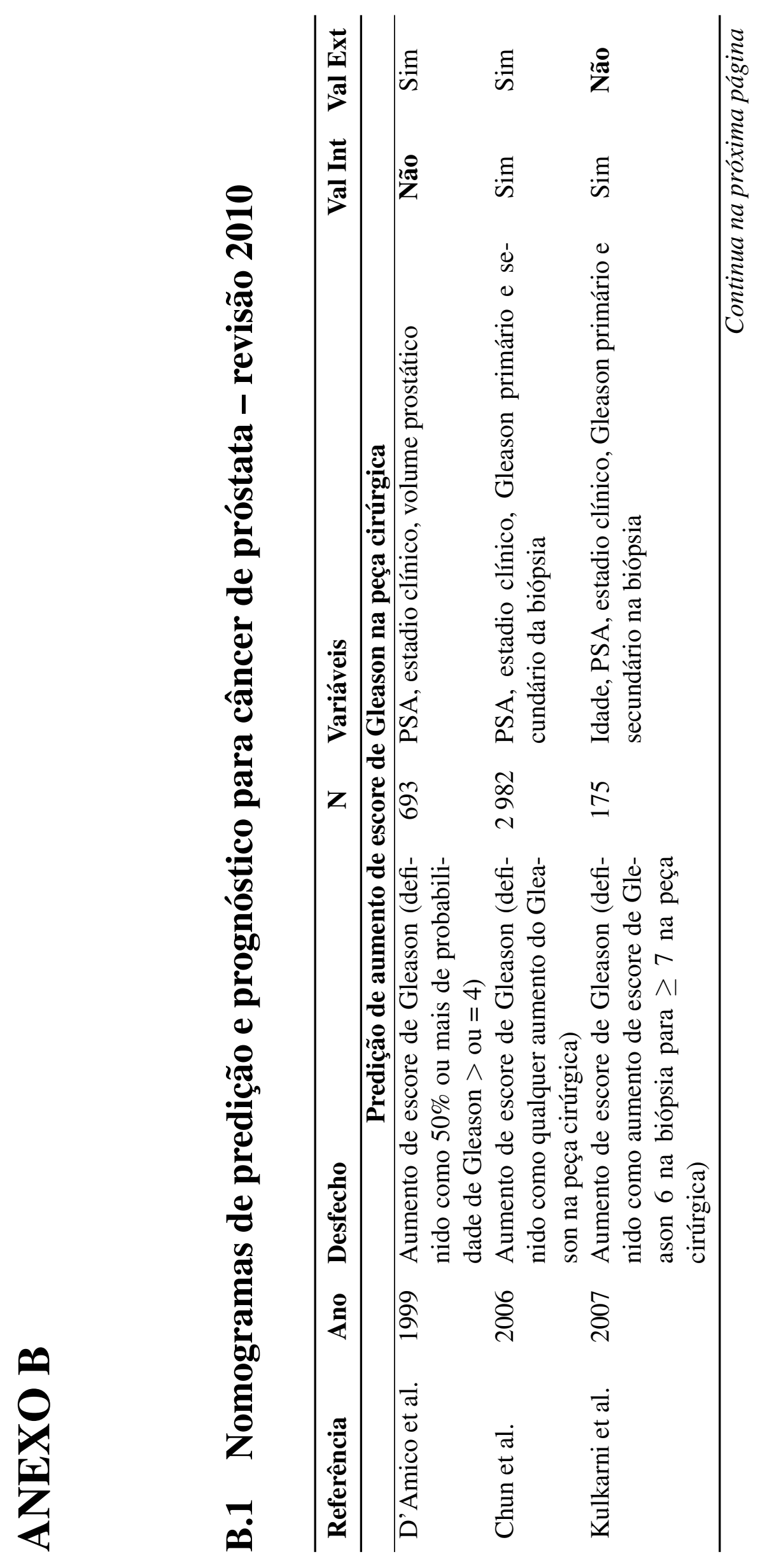




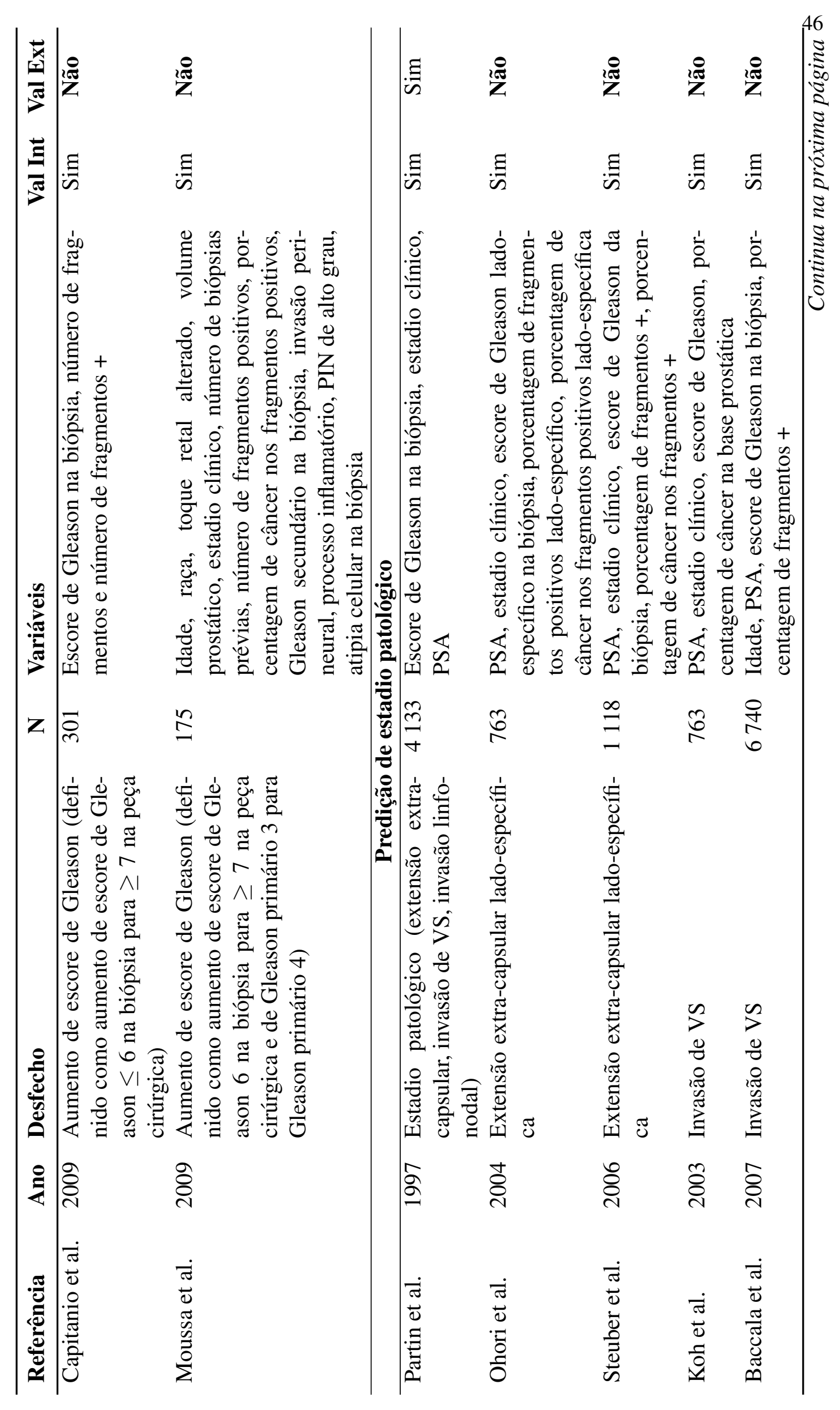




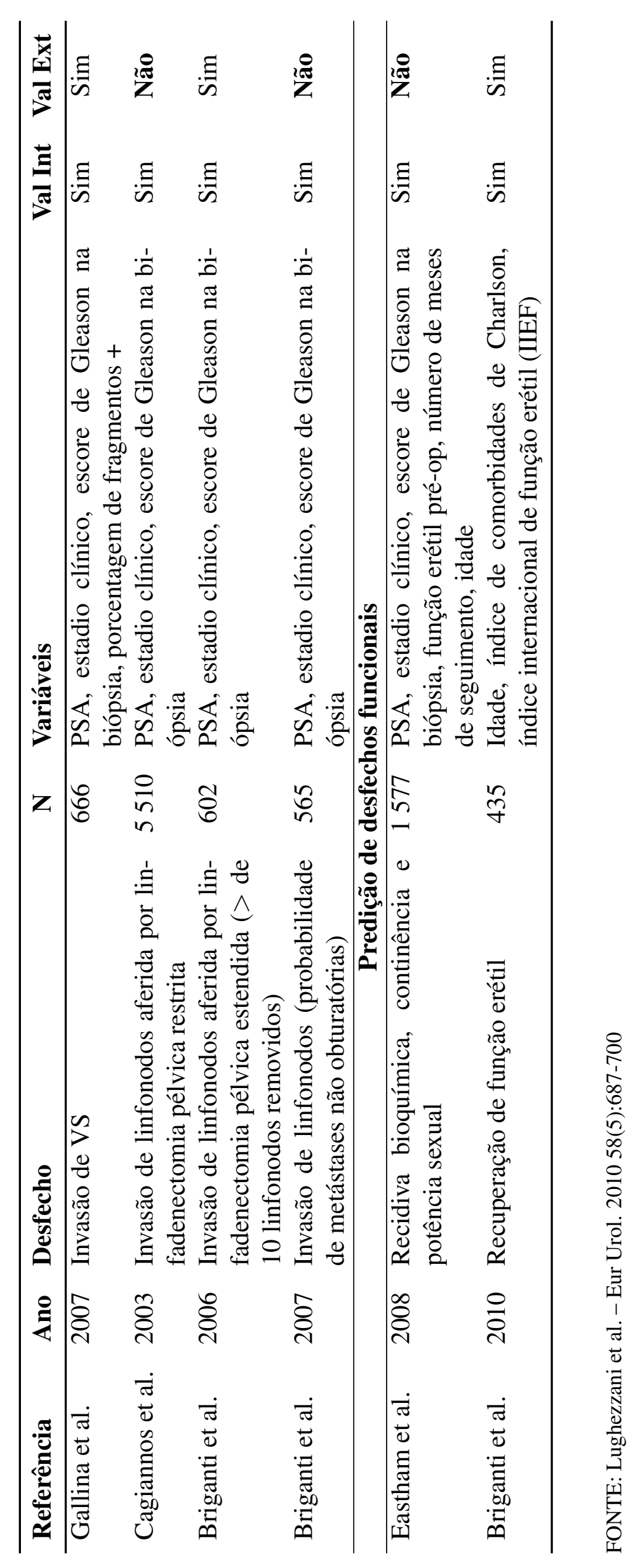




\section{ANEXO C}

\section{C.1 Nomograma 1 de Crippa e Srougi: Predição de doença órgão-confinada de acordo com PSA pré- operatório, escore de Gleason da biópsia e porcen- tagem de fragmentos positivos na biópsia}

\begin{tabular}{lcccc}
\hline \multicolumn{5}{c}{ Gleason 2-6 } \\
PSA \\
\hline PFPB & $\mathbf{0 ~ - 4}$ & $\mathbf{4 , 1 - 1 0}$ & $\mathbf{1 0 , 1 - 2 0}$ & $>\mathbf{2 0}$ \\
$0-25 \%$ & $86[78-93]$ & $80[75-85]$ & $75[68-82]$ & $78[65-87]$ \\
$25,1-50 \%$ & $82[74-90]$ & $75[69-80]$ & $69[61-75]$ & $71[59-82]$ \\
$50,1-75 \%$ & $80[67-90]$ & $72[63-80]$ & $66[55-76]$ & $68[53-82]$ \\
$75,1-100 \%$ & $72[55-85]$ & $59[46-71]$ & $50[37-63]$ & $51[34-67]$ \\
\hline \multicolumn{5}{c}{ Gleason 7 } \\
\multicolumn{5}{c}{ PSA } \\
\hline PFPB & $\mathbf{0 - 4}$ & $\mathbf{4 , 1 - 1 0}$ & $\mathbf{1 0 , 1 - 2 0}$ & $>\mathbf{2 0}$ \\
$0-25 \%$ & $60[55-83]$ & $59[48-69]$ & $51[39-63]$ & $53[35-70]$ \\
$25,1-50 \%$ & $63[50-77]$ & $51[42-60]$ & $42[31-52]$ & $43[28-60]$ \\
$50,1-75 \%$ & $59[43-76]$ & $47[36-59]$ & $39[27-51]$ & $40[25-58]$ \\
$75,1-100 \%$ & $46[29-68]$ & $31[21-44]$ & $22[13-34]$ & $22[12-37]$ \\
\hline \multicolumn{5}{c}{ Gleason 8-9 } \\
\multicolumn{5}{c}{ PSA } \\
\hline PFPB & $\mathbf{0 - 4}$ & $\mathbf{4 , 1 - 1 0}$ & $\mathbf{1 0 , 1 - 2 0}$ \\
$0-25 \%$ & $70[53-84]$ & $59[47-71]$ & $52[38-65]$ & $>\mathbf{2 0}$ \\
$25,1-50 \%$ & $64[46-79]$ & $52[38-64]$ & $43[30-56]$ & $45[27-63]$ \\
$50,1-75 \%$ & $\ldots$ & $48[33-62]$ & $40[25-55]$ & $42[25-61]$ \\
$75,1-100 \%$ & $\ldots$ & $33[20-48]$ & $24[13-38]$ & $24[12-42]$ \\
\hline
\end{tabular}

FONTE: Crippa et al. - Int Braz J Urol - 2006 - 32(2):155-64 
C.2 Nomograma 2 de Crippa e Srougi: Predição de doença órgão-confinada de acordo com PSA préoperatório, escore de Gleason da biópsia e porcentagem de fragmentos positivos na biópsia agrupando escores de Gleason 7-10

\begin{tabular}{lcccc}
\hline \multicolumn{5}{c}{ Gleason 2-6 } \\
PSA & \\
\hline PFPB & $\mathbf{0 - 4}$ & $\mathbf{4 , 1 - 1 0}$ & $\mathbf{1 0 , 1 - 2 0}$ & $>\mathbf{2 0}$ \\
$0-25 \%$ & $86[78-92]$ & $80[75-85]$ & $75[69-82]$ & $78[66-87]$ \\
$25,1-50 \%$ & $82[75-90]$ & $75[70-80]$ & $69[63-76]$ & $71[59-83]$ \\
$50,1-75 \%$ & $80[68-89]$ & $72[63-80]$ & $66[55-75]$ & $68[53-81]$ \\
$75,1-100 \%$ & $72[57-84]$ & $59[47-71]$ & $50[37-62]$ & $51[35-67]$ \\
\hline \multicolumn{5}{c}{ Gleason 7-10 } \\
& \multicolumn{5}{|c}{} & \\
\hline PFPB & $\mathbf{0 - 4}$ & $\mathbf{4 , 1 - 1 0}$ & $\mathbf{1 0 , 1 - 2 0}$ & $>\mathbf{2 0}$ \\
$0-25 \%$ & $70[57-81]$ & $59[50-67]$ & $51[40-62]$ & $54[37-69]$ \\
$25,1-50 \%$ & $64[51-77]$ & $51[43-59]$ & $42[33-52]$ & $44[30-60]$ \\
$50,1-75 \%$ & $59[43-75]$ & $47[36-58]$ & $39[27-51]$ & $41[26-57]$ \\
$75,1-100 \%$ & $47[29-66]$ & $32[21-44]$ & $23[14-33]$ & $23[12-36]$ \\
\hline
\end{tabular}

FONTE: Crippa et al. - Int Braz J Urol - 2006 - 32(2):155-64 


\section{Referências}

1 Lytton B. Prostate cancer: a brief history and the discovery of hormonal ablation treatment. J Urol. 2001;165(6 Pt 1):1859-1862.

2 Adams J. The case of scirrhous of the prostate gland with corresponding affliction of the lymphatic glands in the lumbar region and in the pelvis. Lancet. 1853;1:393.

3 Young HH. The early diagnosis and radical cure of carcinoma of the prostate. Being a study of 40 cases and presentation of a radical operation which was carried out in four cases. 1905. J Urol. 2002;167(2 Pt 2):939-46.

4 Millin T. Retropubic prostatectomy: a new extravesical technique report on 20 cases. 1945. J Urol. 2002;167(2 Pt 2):976-9.

5 Walsh PC, Lepor H, Eggleston JC. Radical prostatectomy with preservation of sexual function: anatomical and pathological considerations. Prostate. 1983;4(5):473-85.

6 Holund B. Latent prostatic cancer in a consecutive autopsy series. Scand J Urol Nephrol. 1980;14(1):29-35.

7 Sakr WA, Haas GP, Cassin BF, Pontes JE, Crissman JD. The frequency of carcinoma and intraepithelial neoplasia of the prostate in young male patients. J Urol. 1993;150(2 Pt 1):379-85.

8 Yin M, Bastacky S, Chandran U, Becich MJ, Dhir R. Prevalence of incidental prostate cancer in the general population: a study of healthy organ donors. J Urol. $2008 ; 179(3): 892-5$.

9 Jemal A, Siegel R, Xu J, Ward E. Cancer statistics, 2010. CA Cancer J Clin. 2010;60(5):277-300.

10 Jemal A, Tiwari RC, Murray T, Ghafoor A, Samuels A, Ward E, et al. Cancer statistics, 2004. CA Cancer J Clin. 2004;54(1):8-29.

11 Albano JD, Ward E, Jemal A, Anderson R, Cokkinides VE, Murray T, et al. Cancer mortality in the United States by education level and race. J Natl Cancer Inst. 2007;99(18):1384-94.

12 Clegg LX, Reichman ME, Miller BA, Hankey BF, Singh GK, Lin YD, et al. Impact of socioeconomic status on cancer incidence and stage at diagnosis: selected findings from the surveillance, epidemiology, and end results: National Longitudinal Mortality Study. Cancer Causes Control. 2009;20(4):417-35. 
13 Quinn M, Babb P. Patterns and trends in prostate cancer incidence, survival, prevalence and mortality. Part II: individual countries. BJU Int. 2002;90(2):174-84.

14 Ferlay J, Shin HR, Bray F, Forman D, Mathers C, Parkin DM. Estimates of worldwide burden of cancer in 2008: GLOBOCAN 2008. Int J Cancer. 2010;127(12):2893-917.

15 Taxas de mortalidade por câncer de Próstata, brutas e ajustadas por idade, pelas populações mundial e brasileira, por 100.000 homens, Brasil, entre 1979 e 2007.; 2011. Available from: http://mortalidade.inca.gov.br/Mortalidade/.

16 Estimativa 2010: incidência de câncer no Brasil; 2010. Available from: http: //www.inca.gov.br/estimativa/2010/.

17 Hugosson J, Aus G, Lilja H, Lodding P, Pihl CG. Results of a randomized, population-based study of biennial screening using serum prostate-specific antigen measurement to detect prostate carcinoma. Cancer. 2004;100(7):1397-405.

18 Määttänen L, Hakama M, Tammela TLJ, Ruutu M, Ala-Opas M, Juusela H, et al. Specificity of serum prostate-specific antigen determination in the Finnish prostate cancer screening trial. Br J Cancer. 2007;96(1):56-60.

19 Thompson IM, Goodman PJ, Tangen CM, Lucia MS, Miller GJ, Ford LG, et al. The influence of finasteride on the development of prostate cancer. N Engl J Med. 2003;349(3):215-24.

20 Vickers AJ, Lilja H. Cutpoints in clinical chemistry: time for fundamental reassessment. Clin Chem. 2009;55(1):15-17.

21 Gleason DF, Mellinger GT, Group VACUR. Prediction of prognosis for prostatic adenocarcinoma by combined histological grading and clinical staging. 1974. J Urol. 2002;167(2 Pt 2):953-8.

22 Antunes AA, Srougi M, Dall'Oglio MF, Crippa A, Paranhos M, Cury J, et al. Microvascular invasion is an independent prognostic factor in patients with prostate cancer treated with radical prostatectomy. Int Braz J Urol. 2006;32(6):668-75.

23 Partin AW, Mangold LA, Lamm DM, Walsh PC, Epstein JI, Pearson JD. Contemporary update of prostate cancer staging nomograms (Partin Tables) for the new millennium. Urology. 2001;58(6):843-8.

24 Kattan MW, Eastham JA, Stapleton AM, Wheeler TM, Scardino PT. A preoperative nomogram for disease recurrence following radical prostatectomy for prostate cancer. J Natl Cancer Inst. 1998;90(10):766-71.

25 D'Amico AV, Whittington R, Malkowicz SB, Schultz D, Fondurulia J, Chen MH, et al. Clinical utility of the percentage of positive prostate biopsies in defining biochemical outcome after radical prostatectomy for patients with clinically localized prostate cancer. J Clin Oncol. 2000;18(6):1164-72. 
26 Peller PA, Young DC, Marmaduke DP, Marsh WL, Badalament RA. Sextant prostate biopsies. A histopathologic correlation with radical prostatectomy specimens. Cancer. 1995;75(2):530-8.

27 D'Amico AV, Whittington R, Malkowicz SB, Schultz D, Blank K, Broderick GA, et al. Biochemical outcome after radical prostatectomy, external beam radiation therapy, or interstitial radiation therapy for clinically localized prostate cancer. JAMA. 1998;280(11):969-74.

28 Touijer K, Scardino PT. Nomograms for staging, prognosis, and predicting treatment outcomes. Cancer. 2009;115(13 Suppl):3107-11.

29 Sokoloff $\mathrm{MH}$, Brendler CB. Indications and contraindications for nerve-sparing radical prostatectomy. Urol Clin North Am. 2001;28(3):535-43.

30 Klotz L. Active surveillance for favorable-risk prostate cancer: who, how and why? Nat Clin Pract Oncol. 2007;4(12):692-8.

31 Lughezzani G, Briganti A, Karakiewicz PI, Kattan MW, Montorsi F, Shariat SF, et al. Predictive and prognostic models in radical prostatectomy candidates: a critical analysis of the literature. Eur Urol. 2010;58(5):687-700.

32 McGinn TG, Guyatt GH, Wyer PC, Naylor CD, Stiell IG, Richardson WS. Users' guides to the medical literature: XXII: how to use articles about clinical decision rules. Evidence-Based Medicine Working Group. JAMA. 2000;284(1):79-84.

33 Crippa A, Srougi M, Dall'Oglio M, Antunes A, Leite K, Nesrallah L, et al. A new nomogram to predict pathologic outcome following radical prostatectomy. Int Braz J Urol. 2006;32(2):155-64.

34 Schröder FH, Hermanek P, Denis L, Fair WR, Gospodarowicz MK, PavoneMacaluso M. The TNM classification of prostate cancer. Prostate Suppl. 1992;4:129-38.

35 Efron B, Tibshirani RJ. An Introduction to the Bootstrap: Monographs on Statistics and Applied Probability, Vol. 57. Chapmann and Hall; 1998.

36 Justice AC, Covinsky KE, Berlin JA. Assessing the generalizability of prognostic information. Ann Intern Med. 1999;130(6):515-24.

37 Janssen KJM, Moons KGM, Kalkman CJ, Grobbee DE, Vergouwe Y. Updating methods improved the performance of a clinical prediction model in new patients. J Clin Epidemiol. 2008;61(1):76-86.

38 Edge SB, Compton CC. The American Joint Committee on Cancer: the 7th edition of the AJCC cancer staging manual and the future of TNM. Ann Surg Oncol. 2010;17(6):1471-4.

39 Gleason DF. Classification of prostatic carcinomas. Cancer Chemother Rep. 1966;50(3):125-8. 
40 Reiner WG, Walsh PC. An anatomical approach to the surgical management of the dorsal vein and Santorini's plexus during radical retropubic surgery. J Urol. 1979;121(2):198-200.

41 Srougi M, Nesrallah LJ, Kauffmann JR, Nesrallah A, Leite KR. Urinary continence and pathological outcome after bladder neck preservation during radical retropubic prostatectomy: a randomized prospective trial. J Urol. 2001;165(3):815-8.

42 Peduzzi P, Concato J, Kemper E, Holford TR, Feinstein AR. A simulation study of the number of events per variable in logistic regression analysis. J Clin Epidemiol. 1996;49(12):1373-9.

43 Harrell FE. Regression Modeling Strategies: With Applications to Linear Models, Logistic Regression, and Survival Analysis. New York: Springer Verlag; 2001.

44 Raaijmakers R, Kirkels WJ, Roobol MJ, Wildhagen MF, Schrder FH. Complication rates and risk factors of 5802 transrectal ultrasound-guided sextant biopsies of the prostate within a population-based screening program. Urology. 2002;60(5):82630 .

45 Simsir A, Kismali E, Mammadov R, Gunaydin G, Cal C. Is it possible to predict sepsis, the most serious complication in prostate biopsy? Urol Int. 2010;84(4):3959.

46 Yu JB, Makarov DV, Sharma R, Peschel RE, Partin AW, Gross CP. Validation of the partin nomogram for prostate cancer in a national sample. J Urol. 2010;183(1):105-11.

47 Bhojani N, Ahyai S, Graefen M, Capitanio U, Suardi N, Shariat SF, et al. Partin Tables cannot accurately predict the pathological stage at radical prostatectomy. Eur J Surg Oncol. 2009;35(2):123-8.

48 Altman DG, Royston P. What do we mean by validating a prognostic model? Stat Med. 2000;19(4):453-73.

49 Partin AW, Kattan MW, Subong EN, Walsh PC, Wojno KJ, Oesterling JE, et al. Combination of prostate-specific antigen, clinical stage, and Gleason score to predict pathological stage of localized prostate cancer. A multi-institutional update. JAMA. 1997;277(18):1445-51.

50 Blute ML, Bergstralh EJ, Partin AW, Walsh PC, Kattan MW, Scardino PT, et al. Validation of Partin tables for predicting pathological stage of clinically localized prostate cancer. J Urol. 2000;164(5):1591-5.

51 Makarov DV, Trock BJ, Humphreys EB, Mangold LA, Walsh PC, Epstein JI, et al. Updated nomogram to predict pathologic stage of prostate cancer given prostatespecific antigen level, clinical stage, and biopsy Gleason score (Partin tables) based on cases from 2000 to 2005. Urology. 2007;69(6):1095-101. 
52 Karakiewicz PI, Bhojani N, Capitanio U, Reuther AM, Suardi N, Jeldres C, et al. External validation of the updated Partin tables in a cohort of North American men. J Urol. 2008;180(3):898-902.

53 Smith DS, Catalona WJ. The nature of prostate cancer detected through prostate specific antigen based screening. J Urol. 1994;152(5 Pt 2):1732-6.

54 Boevee SJ, Venderbos LDF, Tammela TLJ, Nelen V, Ciatto S, Kwiatkowski M, et al. Change of tumour characteristics and treatment over time in both arms of the European Randomized study of Screening for Prostate Cancer. Eur J Cancer. 2010;46(17):3082-9.

55 Sebo TJ, Bock BJ, Cheville JC, Lohse C, Wollan P, Zincke H. The percent of cores positive for cancer in prostate needle biopsy specimens is strongly predictive of tumor stage and volume at radical prostatectomy. J Urol. 2000;163(1):174-8.

56 Steyerberg EW, Vickers AJ, Cook NR, Gerds T, Gonen M, Obuchowski N, et al. Assessing the performance of prediction models: a framework for traditional and novel measures. Epidemiology. 2010;21(1):128-38.

57 Albers P, Schäfers S, Löhmer H, de Geeter P. Seminal vesicle-sparing perineal radical prostatectomy improves early functional results in patients with low-risk prostate cancer. BJU Int. 2007;100(5):1050-4.

58 Jacob R, Hanlon AL, Horwitz EM, Movsas B, Uzzo RG, Pollack A. Role of prostate dose escalation in patients with greater than $15 \%$ risk of pelvic lymph node involvement. Int J Radiat Oncol Biol Phys. 2005;61(3):695-701.

59 Chun FKH, Graefen M, Zacharias M, Haese A, Steuber T, Schlomm T, et al. Anatomic radical retropubic prostatectomy-long-term recurrence-free survival rates for localized prostate cancer. World J Urol. 2006;24(3):273-80.

60 Roemeling S, Roobol MJ, Kattan MW, van der Kwast TH, Steyerberg EW, Schröder FH. Nomogram use for the prediction of indolent prostate cancer: impact on screen-detected populations. Cancer. 2007;110(10):2218-21.

61 Steyerberg EW, Roobol MJ, Kattan MW, van der Kwast TH, de Koning HJ, Schröder FH. Prediction of indolent prostate cancer: validation and updating of a prognostic nomogram. J Urol. 2007;177(1):107-12.

62 Kattan MW, Cuzick J, Fisher G, Berney DM, Oliver T, Foster CS, et al. Nomogram incorporating PSA level to predict cancer-specific survival for men with clinically localized prostate cancer managed without curative intent. Cancer. 2008;112(1):69-74.

63 Lowrance WT, Scardino PT. Predictive models for newly diagnosed prostate cancer patients. Rev Urol. 2009;11(3):117-26. 